\title{
On the relationship between the cosmological background field and the Higgs field
}

\author{
Engel Roza \\ Philips Research Labs, Eindhoven, The Netherlands (retired) \\ Email: engel.roza@onsbrabantnet.nl
}

\section{Summary}

It is shown that the relationship between gravity and quantum physics can be described in terms of the symmetry break of space due to elementary constituents, dubbed as "darks", which constitute a universal energetic background field that extends from the cosmological level down to the nuclear level. It requires (a) the awareness of the polarisable second elementary dipole moment of a recently discovered third Dirac particle type, next to the electron-type and the Majorana-type, and (b) the awareness that Einstein's Lambda is not a constant of nature, but, instead, a covariant integration constant with a value that depends on the scope of the cosmological system under consideration, such as solar systems and galaxies, eventually showing up as the Cosmological Constant at the level of the universe. The relationship has been made explicit by relating two major gravitational constants quantaties (the gravitational constant and Milgrom's acceleration constant) with a single nuclear quantity (the rest mass of the pion derived from the Higgs boson value).

Keywords: Cosmological Constant; Higgs field; Milgrom's constant; Dirac particle; unification

\section{Introduction}

Present-day theory of quantum physics as well as present-day theory of gravity rely upon the presence of an omni-present energetic background field. In quantum physics, this field is known as the "Higgs field". It is required for explaining the origin of mass. It has an axiomatic definition, conceived in 1964,[1]. In gravity, the existence of the background field is required to explain the accelerated expansion of the universe, known since 1998, [2]. This cosmological background field has been defined on the basis of Einstein's Cosmological Constant [3]. It is also known as "dark energy". It would be odd if two different energetic background fields would exist next to each other. More logical would be if the Higgs field and the cosmological background field would be the same. In both cases the unavoidable conclusion is that there is not such a thing as "empty space", but that space is filled with an energetic fluidum. This conclusion has given rise to the idea of conceiving the vacuum as an entropic medium filled with energetic constituents, in this article to be annotated as darks. As long as these darks are not subject to any directional energetic influence, their motions remain fully chaotic. In that state the vacuum is fully symmetric, because its state before and after a time interval of "closed eyes" with an arbitrary translation or rotation of the observer, is just the same [4]. It means that the awareness of a Higgs field and a Cosmological Constant implies a symmetry break, respectively in nuclear space and in cosmological space. This is the issue that will be discussed in this article.

In $[5,6,7,8,9]$ it has been argued that if the cosmological background field would consist of energetic uniformly distributed polarisable vacuum particles, the dark energy would give an explanation for the dark matter problem as well, because vacuum polarization would evoke a gravitational equivalent of the well-known Debije effect [10]. With the difference, though, 
that the central force from a gravitational nucleus is enhanced just opposite to the suppression of the Coulomb force from an electrically charged nucleus in an ionized plasma. This picture fits to the Higgs field of nuclear particle physics as well, albeit that the energetic background particles would show the true Debije effect, in the sense that they would exponentially suppress a central nuclear force such as required to explain the short range of nuclear forces. It corresponds more or less with the common view that mass-less force carrying particles are retarded by a surrounding field of energy, thereby gaining mass $[11,12]$.

The modeling of the omni-present background energy by energetic vacuum particles, requires a model for its elementary constituent (the dark). This element must be a source of energy, and must be force feeling as well. In those aspects it resembles an electron, which is ultimately the source of electromagnetic energy, and which is sensitive to the fields spread by other electrons. However, whereas the dark in the cosmological background field must be polarisable under the gravitational potential, an electron is non-polarisable under an electric potential. The electric dipole moment of an electron is zero, while a dark should have a nonzero gravitational dipole moment. In $[9,13,14]$ the suggestion has been made that these particles could be of the particular Dirac type as theorized back in 1937 by Ettore Majorana [15]. There is, however, no convincing argument why a Majorana particle would have a dipole moment that is polarisable in a scalar potential field. It is recognized, though, that Dirac's theory contains some heuristic elements. Recently, the author of this article found a third type Dirac particle, next to the electron type and the Majorana type [16]. This third has the unique property that, unlike the electron type, it possesses a dipole moment that is polarisable in a scalar potential field. It is my aim to show in this article that this third matches with the dark. In the next paragraph first a summary will be given of the third. Thereafter a view will be given on the cosmological background energy and its impact on gravity.

The cosmological and gravity view to be developed in this article relies, next to the awareness of the darks, on a particular interpretation of the $\Lambda$ parameter in Einstein's Field Equation. Different from the common perception that Einstein's $\Lambda$ is a constant of nature, usually identified as the Cosmological Constant, it is in the author's view a covariant integration constant that may have different values depending on the scope of a cosmological system under consideration. Because it may depend on other attributes but just time-space coordinates, such as mass content, for instance, it may have different values at the level of solar systems, galaxies and the universe. Only at the latter level, it is justified to identify the $\Lambda$ as the Cosmological Constant indeed. At that level, by the way, the cosmological system is in a state of maximum symmetry and maximum entropy. The viability of this view will be proven by a calculation of Milgrom's empirical acceleration constant of dark matter.

After that, it will be shown that the novel Dirac particle applies to quarks as well, ending up in a model for the nuclear domain, in which the common Lagrangian description of the Higgs field is harmonized with a nuclear energetic background field with similar characteristics as the cosmological one. Finally it is shown in verifiable formulae how these fields are related. 


\section{Summary of the third}

The canonic formulation of Dirac's particle equation reads as $[17,18]$,

$\left(\mathrm{i} \hbar \gamma^{\mu} \partial_{\mu} \psi-\beta m_{0} c \psi\right)=0$.

In which $\beta$ is the $4 \times 4$ unity matrix and in which the $4 \times 4$ gamma matrices have the properties,

$\gamma_{\mu} \gamma_{\nu}+\gamma_{\nu} \gamma_{\mu}=0$ if $\mu \neq v$; and $\gamma_{0}^{2}=1 ; \gamma_{i}^{2}=-1$

As usual, $c$ is the vacuum light velocity, $\hbar$ is the reduced Planck constant and $m_{0}$ is the rest mass of the particle. Whereas the canonical set is given by,

$\gamma_{0}=\left[\begin{array}{cc}I & 0 \\ 0 & -I\end{array}\right] ; \gamma_{1}=\left[\begin{array}{cc}0 & \sigma_{1} \\ -\sigma_{1} & 0\end{array}\right] ; \gamma_{2}=\left[\begin{array}{cc}0 & \sigma_{2} \\ -\sigma_{2} & 0\end{array}\right] ; \gamma_{3}=\left[\begin{array}{cc}0 & \sigma_{3} \\ -\sigma_{3} & 0\end{array}\right] ; \beta=\left[\begin{array}{cc}I & 0 \\ 0 & I\end{array}\right]$,

the $\gamma$-set of the third type has been found as [16],

$\gamma_{0}=\left[\begin{array}{cc}0 & -I \\ -I & 0\end{array}\right] ; \gamma_{1}=\left[\begin{array}{cc}\mathrm{i} \sigma_{1} & 0 \\ 0 & -\mathrm{i} \sigma_{1}\end{array}\right] ; \gamma_{2}=\left[\begin{array}{cc}\mathrm{i} \sigma_{2} & 0 \\ 0 & -\mathrm{i} \sigma_{2}\end{array}\right] ; \gamma_{3}=\left[\begin{array}{cc}\mathrm{i} \sigma_{3} & 0 \\ 0 & -\mathrm{i} \sigma_{3}\end{array}\right] ; \beta=\left[\begin{array}{cc}0 & I \\ -I & 0\end{array}\right]$,

in which $\sigma_{i}$ are the Pauli matrices. Besides, the set is subject to an additional constraint, because instead of (1a),

$\gamma_{\mu} \gamma_{v}+\gamma_{v} \gamma_{\mu}=0$ if $\mu \neq v ; \gamma_{\mu} \beta+\beta \gamma_{\mu}=0$ and $\gamma_{0}^{2}=1 ; \gamma_{i}^{2}=-1 ; \beta^{2}=1$

Although the wave equations of the electron type and that of the "third" are hardly different, there is a major difference in an important property. Both have two dipole moments. A first one, to be indicated in this text as the first dipole moment, is associated with the elementary angular momentum $\hbar$. The second one, to be indicated as the second dipole moment is associated with the vector $\hbar / c$. These dipole moments show up in the calculation of the excess energy of the particle in motion subject to a vector potential $\boldsymbol{A}\left(A_{0}, A_{x}, A_{y}, A_{z}\right)$. In the canonic case (2a) we have,

$\Delta E=\frac{e \hbar}{2 m_{0}}\left[\begin{array}{cc}\bar{\sigma} \cdot \mathbf{B} & 0 \\ 0 & \bar{\sigma} \cdot \mathbf{B}\end{array}\right]+\frac{e \hbar}{2 m_{0} c}\left[\begin{array}{cc}0 & \mathrm{i} \bar{\sigma} \cdot \mathbf{E} \\ \mathrm{i} \bar{\sigma} \cdot \mathbf{E} & 0\end{array}\right]$

in which $\bar{\sigma}$ is the Pauli vector, defined by

$\bar{\sigma}=\sigma_{1} \mathbf{i}+\sigma_{2} \mathbf{j}+\sigma_{3} \mathbf{k}$

in which ( $\mathbf{i}, \mathbf{j}, \mathbf{k})$ are the spatial unit vectors and in which $\mathbf{B}$ and $\mathbf{E}$ are field vectors derived from the vector potential. The redundancy in (3) allows writing it as, 
$\Delta E=\frac{e}{2 m_{0}}(\bar{\sigma} \hbar \cdot \mathbf{B}+\mathrm{i} \bar{\sigma} \hbar / \mathbf{c} \cdot \mathbf{E})$

The electron has a real first dipole moment $\left(e \hbar / 2 m_{0}\right)$, known as the magnetic dipole moment, and an imaginary second dipole moment (ieh/2 $m_{0} c$ ), known as the anomalous electric dipole moment. The spin vector $S=\bar{\sigma} / 2$ has an eigen value $|\boldsymbol{S}|=1 / 2$. In the case that the Dirac particle is of the third type, as defined by (2b), we have [16],

$$
\Delta E=\frac{e}{2 m_{0}}(\bar{\sigma} \hbar \cdot \mathbf{B} \pm \bar{\sigma} \hbar / \boldsymbol{c} \cdot \mathbf{E})
$$

Figure 1 illustrates the difference between the electron type and the third.

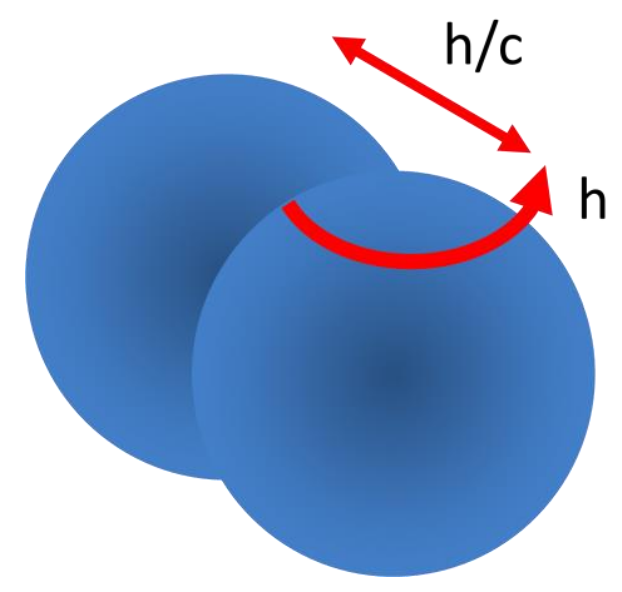

Fig. 1. Like all Dirac particles, the dark has two anomalous dipole moments, a first one due to an elementary angular momentum $\hbar$ and a second one due to an elementary linear momentum $\hbar / c$. While in the case of an electron the latter one is a dynamic one with a zero static value, it is a static one in the case of a dark.

This third type Dirac particle has two real dipole moments, generically without identifying it as an electromagnetic one, to the amounts of $\bar{\sigma} \hbar$, respectively $\bar{\sigma} \hbar / c$. If the dark would be of the electron type, it would not be polarisable in a gravitational field, because such a field is Coulomb-like and is unable to polarize an imaginary second dipole moment. If, however, the dark is a third type, its second dipole moment can be polarized under influence of a scalar potential field. This field is not necessarily the electromagnetic one. The coupling factor is not necessarily the elementary electric charge. If the field is just a static one, eq. (5) can be written as,

$$
\Delta E=-\frac{g \bar{\sigma}}{2 m_{0}}\left(\hbar / c \cdot \nabla A_{0}\right)
$$

In which $g$ is a generic coupling factor. Hence, taking into account that the eigen value of the spin vector with the state variable $\bar{\sigma}$ is $|\boldsymbol{S}|=|\bar{\sigma} / 2|=1 / 2$, the dipole moment $p$ of a single particle in a gravity field (where $g=m_{0}$ ), is given by, 
$p=\frac{\hbar}{2 c}$

Hence, the third type is a candidate for being the elementary constituent of the cosmological background energy. Further profiling of this constituent will be given in the next paragraph.

\section{The cosmological background field}

The presence of an omnipresent background field is imposed by the vacuum solution of Einstein's Field Equation with Einstein's (cosmological) constant $\Lambda,[19,20]$. This Field Equation reads as,

$G_{\mu \nu}+\Lambda g_{\mu v}=\frac{8 \pi G}{c^{4}} T_{\mu \nu} ; G_{\mu \nu}=R_{\mu \nu}-\frac{1}{2} R g_{\mu \nu}$,

in which $T_{\mu \nu}$ is the stress-energy tensor, which describes the energy and the momenta of the source(s) and in which $R_{\mu v}$ and $R$ are, respectively, the so-called Ricci tensor and the Ricci scalar. These can be calculated if the metric tensor components $g_{\mu \nu}$ are known [21,22]. In the case that a particle under consideration is subject to a central force only, the time-space condition shows a spherical symmetric isotropy. This allows to read the metric elements $g_{i j}$ from a simple line element that can be written as

$\mathrm{d} s^{2}=g_{t t}(r, t) \mathrm{d} q_{0}^{2}+g_{r r}(r, t) \mathrm{d} r^{2}+r^{2} \sin ^{2} \vartheta \mathrm{d} \varphi^{2}+r^{2} \mathrm{~d} \vartheta^{2}$

In which $q_{0}=\mathrm{i} c t$ and $\mathrm{i}=\sqrt{-1}$.

Note: The author of this article has a preference for the "Hawking metric" $(+,+,+,+)$ for (ict, $x, y, z$ ), like, for instance, also used by Perkins [23]. By handling time as an imaginary quantity instead of a real one, the ugly minus sign in the metric $(-,+,+,+)$ disappears owing to the obtained full symmetry between the temporal domain and the spatial one.

It means that the number of metric elements $g_{i j}$ reduces to a few, and that only two of them are time and radial dependent. With inclusion of the constant $\Lambda$, a wave equation for a particle under central force can be derived from (8-9) as

$-\frac{\partial^{2} g_{t t}}{c^{2} \partial t^{2}}+\frac{1}{r} \frac{\partial^{2}\left(r g_{t t}\right)}{\partial r^{2}}+2 \Lambda=-g_{r r} \frac{8 \pi G T_{t t}}{c^{2}} \delta^{3}(r) U(t)$.

If $T_{t t}$ were a pointlike source $T_{t t}=M c^{2} \delta^{3}(r) U(t)$, in which $U(t)$ is Heaviside's step function, the static solution of this equation would be provided by the Schwarzschild-de Sitter metric, also known as Kottler metric, $[24,25,26,27]$. Unfortunately, (10) does not allow deriving a meaningful wave equation. Taking the view that the vacuum is something else but empty space, makes a difference. Whereas an empty space with $\Lambda=0$ corresponds with virtual 
sources $T_{\mu \mu}=0$, the vacuum with $\Lambda \neq 0$ is a fluidal space with virtual sources $T_{\mu \mu}=-p \Lambda$, with $g_{\mu \mu}=\left(1,1, r^{2} \sin ^{2} \vartheta, r^{2}\right)$, in which $p=c^{4} / 8 \pi G[28,29,30]$. (Owing to the Hawking metric, $p$ is equal for all diagonal elements). This particular stress-energy tensor, with equal diagonal elements, corresponds with the one for a perfect fluid in thermodynamic equilibrium [22]. Inserting a massive source in this fluid will curve the vacuum to $g_{\mu \mu}=\left(g_{t t}, g_{r r}, r^{2} \sin ^{2} \vartheta, r^{2}\right)$. This allows rewriting (10) as,

$$
-\frac{\partial^{2}}{c^{2} \partial t^{2}}(r \Phi)+\frac{\partial^{2}}{\partial r^{2}}(r \Phi)+\lambda^{2}(r \Phi)=-r \frac{8 \pi G M}{c^{2}} \delta^{3}(r) U(t),
$$

In which $\lambda^{2}=2 \Lambda$.

Note: The linearization of Einstein's highly non-linear Field Equation (8) toward a linear single component wave equation (11) is not trivial. While in the case of $\Lambda=0$ the spherical symmetric metric (9) allows a straightforward derivation of the linear Poisson equation, the derivation of (11) is cumbersome. It can only be done under constraining the range of validity. The validity of this equation is restricted between a low spatial limit $r_{L}$ and a high spatial limit $r_{H} \lambda \approx 6$. It can be found in ref. [31], as well as in the Appendix of this article.

The static format of the wave equation (11) is a potential field set up by a pointlike source with a format that shows up as a modification of Poisson's equation, such that

$$
\frac{\partial^{2}}{\partial r^{2}}(r \Phi)+\lambda^{2}(r \Phi)=-r \frac{8 \pi G M}{c^{2}} \delta^{3}(r)
$$

Under positive space-time curving, (12) can be solved by

$$
\Phi=\Phi_{0} \frac{\cos \lambda r+\sin \lambda r}{\lambda r} ; \Phi_{0}=M G \lambda
$$

Applied to galaxies with effective central mass $M$, this solution gives a fit with Milgrom's empirical enhanced gravity law for, [31],

$$
\lambda^{2}=\frac{2}{5} \frac{a_{0}}{M G} \rightarrow M \lambda^{2}=\frac{2}{5} \frac{a_{0}}{G}
$$

in which $a_{0}$ is Milgrom's acceleration constant that characterizes the dark matter effect. The fit is illustrated in figure 2 . It shows the relative increase of the gravitational acceleration $g$ relative to the Newtonian one $g_{N}$ as a function $\lambda r$. The fit holds to about $r \lambda \approx 2$. This is lower than the upper limit $r_{H} \lambda \approx 6$ that limits the validity range of (13), but, as discussed later, still higher than the radius of Milky Way-type galaxies. 


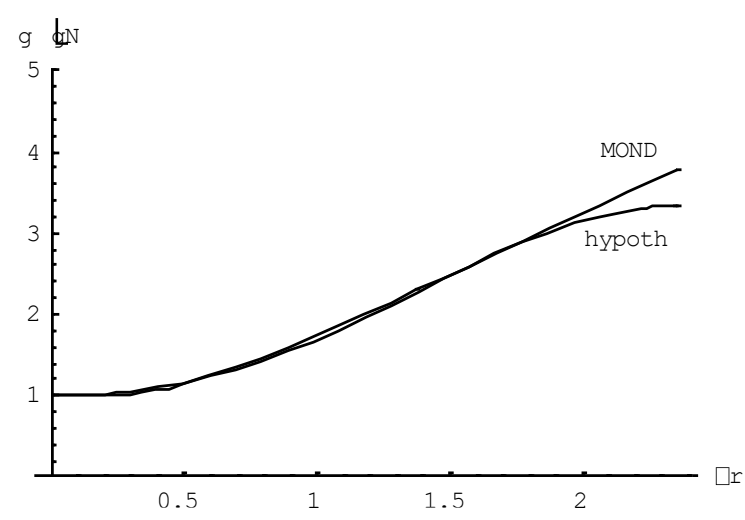

Fig.2 : MOND's interpolation function compared with the theory as developed.

Like shown in the Appendix, the lower limit $r_{L}$ of the validity range is constrained by,

$r_{L} \gg R_{G}$, where $R_{G}=\left(2\left|R_{S}\right| \sqrt{\frac{a_{L}}{a_{0}}} \sqrt{\frac{5 R_{S} L}{2}}\right)^{1 / 2}$,

In which $R_{S}$ is the Schwarzschild radius of the galaxy, $L=c t_{H}, a_{L}=c / t_{H}, t_{H}$ is Hubble time ( $\approx 13.8$ Gyear). The Schwarzschild radius $R_{S}$ of a typical galaxy, like the Milky Way, is about 0.2 lightyear. Milgrom's acceleration constant amounts to $a_{0} \approx 1.2510^{-10} \mathrm{~m} / \mathrm{s}^{2}$. For such a galaxy, the range $R_{G}$ calculated from (14a) appears being $R_{G} \approx 278$ lightyear. Apart from the lower limit, care has to be taken for the upper limit $r_{H}$. From (14)

$r_{H}=\frac{2}{\lambda}=2 \sqrt{\frac{2 a_{0}}{5 M G}}=2 \sqrt{\frac{4}{5} \frac{a_{0}}{c^{2}} \frac{c^{2}}{2 M G}}=2 \sqrt{\frac{4}{5} \frac{a_{0}}{c^{2}} R_{S}}=2 \sqrt{\frac{4}{5} \frac{a_{0}}{a_{L}} R_{S} L} ; a_{L}=\frac{c^{2}}{L} \rightarrow$

$r_{H}($ Milky Way) $\approx 485000$ lightyear.

Considering that the radius of the Milky Way is estimated as 100.000-180.000 lightyear and that our solar system is at about 26.000 lightyear from the center, it will be clear that the wave equation (12) holds for the major part of the Milky Way, and probably all galaxies, thereby solving the anomaly problem of the stellar rotation problem.

Eq. (14) relates the anti-decay parameter $\lambda$ with the mass of a galaxy. Because $\lambda^{2}=2 \Lambda$ as shown by (11), and because $a_{0}$ has appeared being a constant, Einstein's $\Lambda$ is an integration constant with a value that is galaxy dependent. The $\Lambda$ term is missing in Einstein's paper of 1916, in spite of his awareness that he equated an integration constant as zero (see footnote on p.804 in ref. [20]). Later, in 1917, Einstein added this quantity as a covariant integration constant for allowing vacuum solutions of his Field Equation [28,32]. It is usually presently taken for granted that this Lambda is a Cosmological Constant that can be regarded as a constant of nature. In fact, however, it is just a constant in the sense that its value does not depend on space-time coordinates. Hence, it may have different values depending on the scope of a cosmological system under consideration, because it may depend on attributes, 
like mass content for instance. It may have different values at the level of solar systems, galaxies and the universe.

It has to be emphasized here that whereas, up to a certain radial limit, (14) expresses the feature of flat rotation curves of gravitational objects in galaxies, it does not give a numerical value for $a_{0}$. It would be possible if we would know a relationship between $\lambda$ and $M$. In fact, it is known at the level of the universe, because as noted before the vacuum is fluidal space with virtual sources $T_{\mu \mu}=-p \Lambda$, in which $p=c^{4} / 8 \pi G$. Denoting the gravitational background energy density as $\rho$, we have

$\rho c^{2}=\frac{c^{4}}{8 \pi G} \Lambda$

As proven in [31], by conceiving the visible universe as a sphere with distributed matter, in which the matter consists of distributed galaxies, it appears possible to express of Milgrom's acceleration constant in terms of the baryonic content $\Omega_{B}$ as,

$a_{0}=\frac{15}{4} \Omega_{B} a_{L}$

Inserting the empirical $\Lambda \mathrm{CDM}$ value $\Omega_{B}=0.0486$ into this expression gives the well known value $a_{0} \approx 1.25 \times 10^{-10} \mathrm{~m} / \mathrm{s}^{2}$. This gives strong support for the viability of the theory as developed so far. Note that, In spite of now having obtained by theory testable numerical values for dark matter, the true physical nature of it still has remained unclear.

Hence, the issue to be addressed next Is the question whether it is possible to give a physical profile to the constituents of dark matter. Let us conceive these constituents as vacuum particles of the type as described in paragraph 2, i.e., as darks that show a polarizable dipole moment in a scalar potential field. Let us try to establish a density expression for these vacuum particles (darks). To do so, let us rewrite (12) as,

$\nabla^{2} \Phi+\lambda^{2} \Phi=-\frac{4 \pi G M}{c^{2}} \delta^{3}(r)$,

and subsequently into,

$\nabla^{2} \Phi=-4 \pi G \rho(r)$, in which

$\rho(r)=\frac{M}{c^{2}} \delta^{3}(r)+\rho_{D}(r) ; \rho_{D}(r)=\frac{\lambda^{2}}{4 \pi G} \Phi(r)$.

In Debije's theory of electric dipoles [7,10,33,34],

$\rho_{D}(r)=-\nabla \cdot \mathbf{P}_{\mathbf{g}}$.

The vector $\mathbf{P}_{\mathrm{g}}$ is the dipole density. From (21), 
$\rho_{D}=\frac{1}{r^{2}} \frac{\mathrm{d}}{\mathrm{d} r}\left\{r^{2} P_{g}(r)\right\}$

Assuming that in the static condition the space fluid is eventually fully polarized by the field of the pointlike source, $P_{g}(r)$ is a constant $P_{g 0}$. Hence, from (22),

$\rho_{D}(r)=2 \frac{P_{g 0}}{r}$

Taking into account that to first order,

$\Phi(r)=\frac{M G}{r}$,

we have from (23) and (24),

$\rho_{D}(r)=\frac{2 P_{g 0}}{M G} \Phi(r)$

Hence, from (14), (20) and (23-25),

$P_{g 0}=\frac{a_{0}}{20 \pi G}$

Under consideration of the elementary dipole moment $p=h / 2 c$ to the dark, the volume density $N / \mathrm{m}^{3}$ of the darks is found as,

$N / \mathrm{m}^{3}=\frac{a_{0}}{20 \pi G} \frac{2 c}{\hbar}$.

Because the dipole moment is a state variable that can only assume two values, any of the darks resemble an elementary amount of information. Hence, the amount of information or entropy $S(V)$ enclosed in a black hole with volume $V$ amounts to,

$S(V)=\frac{a_{0}}{20 \pi G} \frac{2 c}{\hbar} V$.

Let us consider this result in relation with the Bekenstein-Hawking expression for the entropy of a black hole, which reads as [35,36],

$S_{H}=k_{B} \frac{c^{3}}{4 G \hbar} A$ 
in which $A$ is the black hole's peripheral area and $k_{B}$ is Boltzmann's constant. The peripheral area of a spherical black hole is determined by its Schwarzschild radius as,

$$
A=4 \pi R_{S}^{2}, R_{S}=\frac{2 M G}{c^{2}}
$$

in which $M$ is the baryonic mass of the black hole. Boltzmann's constant shows up as a consequence of the thermodynamic definition of entropy. In that definition $S_{H}$ is not dimensionless, because of the thermodynamic interpretation of entropy as a measure for the unrest of molecules due to temperature, which relates the increase $\Delta S$ of entropy with an increase molecular energy $\Delta E$ due to temperature $T$, such as expressed by the thermodynamic definition,

$\Delta E=T \Delta S$

Boltzmann's famous conjecture connects entropy with information, by stating

$S_{B}=k_{B} \log (\#$ microstates $)$.

This conjecture expresses the expectation that entropy can be expressed in terms of the total number of states that can be assumed by an assembly of molecules. Boltzmann's constant shows up to correct for dimensionality. I would like to emphasize here that (29) and (31) are different definitions for the entropy $S$, and not necessarily identical. Knowing that (29) has been derived from (30) and accepting Boltzmann's conjecture, we would have,

$\frac{c^{3}}{4 G \hbar} A=\log (\#$ microstates $)$.

Both sides of this expression are dimensionless. Omitting Boltzmann's constant makes entropy a dimensionless measure of information, which, of course, is very appealing. At this point, I wish to elaborate on a subtlety, which has been shown by Verlinde. According to Boltzmann's conjecture, an elementary step $\Delta S$ in entropy would imply $\Delta S=k_{B}$. Verlinde has proven, however, that an elementary step in entropy from the Hawking-Bekenstein entropy implies $\Delta S=2 \pi k_{B}$. If not, the Hawking-Bekenstein's formula would violate Newton's gravity law [35]. Because Boltzmann's expression is a conjecture without proof, the problem can be settled by modifying the dimensionless expression of entropy (32) into,

$S=\frac{1}{2 \pi} \frac{c^{3}}{4 G \hbar} A=\log (\#$ microstates $)$

Considering the well-known relationship between the event horizon $c t_{H}$ of the visible universe and the Schwarzschild radius from the critical mass enclosed within that horizon ( $t_{H}$ is the Hubble time scale) that allows conceiving the visible universe as a virtual black hole [37], the entropy within the event horizon of the universe can be established from (28) as, 


$$
S=\frac{a_{0}}{20 \pi G} \frac{2 c}{\hbar} V=\frac{a_{0}}{20 \pi G} \frac{2 c}{\hbar}\left(\frac{4}{3} \pi c^{3} t_{H}^{3}\right),
$$

while we have from (33) under consideration that the baryonic matter is an $\Omega_{B}$ fraction of total matter in the universe,

$$
S=\frac{1}{2 \pi} \frac{c^{3}}{4 G \hbar} \Omega_{B} 4 \pi\left(c t_{H}^{2}\right)
$$

Equating (34) with (35) gives,

$$
a_{0}=\frac{15}{4} \Omega_{B} \frac{c}{t_{H}}
$$

which is just the same as (18).

Hence, it is fair to conclude that the energetic background fluid in the universe is built up by quantum particles. These quantum particles have a polarisable dipole moment $p$ with an eigen value to the amount of $p=\hbar / 2 c$. The volume density of these quantum particles as calculated from (27) amounts to $\mathrm{N} / \mathrm{m}^{3}=1.7 \times 10^{41}$ or, equivalently, $1.7 \times 10^{14}$ particles per cubic nanometer. This makes the energetic background fluid rather smooth. From this volume density and the critical matter density of the universe the mass of these particles can be calculated.

The critical mass density $\rho_{c}$ can be expressed in terms of Hubble time $t_{H}$ from the consideration that the universe is a bubble from which light cannot escape. Hence as a black hole with radius $R_{S}=c t_{H}$, such that

$$
R_{S}=c t_{H}=\frac{2 M_{c} G}{c^{2}} \rightarrow c t_{H}=\frac{2 G}{c^{2}} \frac{4}{3} \pi\left(c t_{H}\right)^{3} \rho_{c} \rightarrow \rho_{c}=\frac{3}{8 \pi G c^{2}}\left(\frac{c}{t_{H}}\right)^{2}
$$

It gives $9.410^{-27} \mathrm{~kg} / \mathrm{m}^{3}$. Divided over $1.7 \times 10^{41}$ particles, it gives a mass of $5.5510^{-68} \mathrm{~kg}$ per particle, which corresponds to a massive energy of $310^{-32} \mathrm{eV}$. This makes the darks virtually mass less.

A more extensive analysis of the cosmological background energy can be found in the preprint "On the symmetry of the universe" [31]. The summary given in this paragraph illustrates that the awareness of the polarisable dipole moment of an elementary particular Dirac particle in a scalar potential field and the awareness that Einstein's Lambda is a generic space-time independent covariant integration constant enables the derivation Milgrom's acceleration constant as a simple expression in terms of Hubble time $t_{H}(\approx 13.8 \mathrm{Gyear}$ ) and the relative baryonic content $\Omega_{B}(\approx 0.0486)$ of the visible universe as shown by (36). 


\section{The nuclear background energy}

Let us proceed trying to set up a similar model for the nuclear background energy similar to the cosmological background energy. The model as developed so far has been based upon a classical field theory. One of the issues to cope with are the different semantics of the field concept in classical physics, in quantum mechanical physics and in particle physics. In classical physics the field is the static solution of an energetic wave equation. The field has an energetic interpretation. In quantum mechanics the field is a solution of Schrödinger's equation, which in fact is the non-relativistic limit of Dirac's equation. This field has an probabilistic interpretation, because its integrated squared value is considered as the probability that a particle is at some moment in some spatial position. In particle physics theory the two fields are unified in a single concept: the quantum field. This is done on the basis of (second) quantization. This allows the description of processes that are subject to an interchange between matter and energy, such as occur in decay processes and scattering processes, including, for instance, recoil. This Quantum Field Theory (QFT) is one of the pillars of the Standard Model of particle physics. Other pillars are the $U(1) \otimes S U(2) \otimes S U(3)$ gauge formalism, electroweak unification, Quantum Chromo Dynamics (QCD) and the mass generating Spontaneous Symmetry Breaking (SSB) of the energetic background field into the Higgs field. Only some of these aspects are within the scope of this article. Because interchange between matter and energy is beyond the scope, the field view is either classical, formalized as $\Phi$, or quantum mechanical, formalized as a multi-component spinor $\Psi\left(\psi_{\mu}\right)$ eventually reduced to a single component $\psi$.

Let us start from a classical view on the most fundamental nuclear particle: the quark. And let us suppose, as usual by the way, that a quark is a Dirac particle [38]. In this text, however, under the assumption that a quark is a Dirac particle of the third type, the second dipole moment of which is polarisable. Let us proceed by profiling a quark as an energetic particle that, similarly as an electron, spreads a scalar field. Let this field $\Phi$ be characterized by a Lagrangian density with the format

$$
\mathcal{L}=-\frac{1}{2} \partial_{\mu} \Phi \partial^{\mu} \Phi+U(\Phi)+\rho \Phi
$$

in which $U(\Phi)$ is the potential energy of an energetic background field and in which $\rho \Phi$ is the source term. Note that this Lagrangian is an energetic one, from which, by application of the Euler-Lagrange equation, an energetic wave equation can be derived. If the background field would have the format,

$U(\Phi)=U_{D B}=\lambda_{D B}^{2} \frac{\Phi^{2}}{2}$

the stationary solution of the wave equation, derived by application of the Euler-Lagrange equation from the Lagrangian, would be 
$\Phi_{D B}=\Phi_{0} \frac{\exp \left(-\lambda_{D B} r\right)}{\lambda_{D B} r}$

Such a field (39) is of a type as described by Debije [10] for a charged particle in an ionic plasma. The wave equation associated with this Lagrangian $(38,39)$ has the same format as the Klein-Gordon equation, which originally has been erroneously conceived as a relativistic extension of the Schrödinger one. In present canonical field theory, this equation is considered as the field equation for a massive spin-zero particle. In a formal way, one might say that a background field of energy has given mass to a mass less boson. A more physical interpretation is the parallel with the scalar field expression for a charged particle in an ionic plasma, In the Debije's theory, it is the influence of the polarized ionic plasma composed by elementary dipoles that shields the mass less boson field from the electric charged pointlike source.

Similarly as an electron, the quark has an energetic monopole, represented by the source term $\rho \Phi$ in the Lagrangian (38). For an electron, the monopole is an electric point charge. For the quark it is the nuclear equivalent of the electric point charge. Next to the monopole, the electron and the quark have two dipole moments. These dipole moments are the results from the elementary angular momentum $\hbar$ and the elementary mass dipole moment $\hbar / c$. In the case of an electron, these dipole moments give rise to, respectively, a real magnetic dipole and an imaginary electric dipole. In the case of a quark, these dipole moments give rise to, respectively, a real equivalent of the magnetic dipole and a real nuclear equivalent of the electric dipole. While, due to its imaginary value, the electric dipole moment of the electron cannot be polarized in a scalar potential field, the nuclear equivalent can, because of its real value.

Similarly as the monopole of the electron, the monopole of the quark, spreads a scalar potential field. This field is able to polarize the electric dipole equivalent of another quark. Such a dipole spreads an en energetic potential with $x^{-2}$ dependency along the orientation axis of the dipole. As a consequence, an equilibrium of forces can arise between a repelling force from the $r^{-1}$ monopole field dependency and the attractive force with $x^{-2}$ dipole field dependency from suitably aligned dipoles of two quarks. Because nuclear forces have a short range, these potential fields must experience a shielding effect akin to the shielding of the field of an electric point charge in an ionized plasma. This shielding is known as the Debije effect. It occurs under influence of an omni-present fluidal field of energy. Hence, in qualitative terms, the potential field of a quark along the axis of the polarisable dipole, can be expressed as,

$\Phi(\lambda x)=\Phi_{0} \exp (-\lambda x)\left\{\frac{1}{(\lambda x)^{2}}-w \frac{1}{\lambda x}\right\}$

in which $\lambda$ (with dimension $\mathrm{m}^{-1}$ ) is a measure for the range of the nuclear potential, in which $\Phi_{0}$ (in units of energy, i.e. joule) is a measure for the quark's "charge", and in which $w$ is a dimensionless weigh factor that relates the strength of the monopole field to the dipole field. The far field, decaying as $\exp (-\lambda x) / \lambda x$ is due to the monopole. As will be shown later, It can be seen as the weak interaction between the quarks. The near field, decaying as 
$\exp (-\lambda x) /(\lambda x)^{2}$ is due the polarisable dipole. While the spatial Debije format (40) of the field has been straightforwardly calculated from the functional expression of the background field (39), the spatial field expression (41) of the quark's field has only been derived indirectly. One may ask if it would be possible to arrive at the format (41) analytically from a functional field expression as well. Obviously, the simple unbiased symmetric background field expression (39), in which $U(\Phi)=0$ for $\Phi=0$, has to be modified for the purpose. The most simple approach is modifying (39) into

$U(\Phi)=-\frac{\mu_{H}^{2}}{2} \Phi^{2}+\frac{\lambda_{H}^{2}}{4} \Phi^{4}$

For positive values of $\lambda_{H}^{2}$ and $\mu_{H}^{2}$, it is a broken field that is zero for $\Phi_{0}=\left(\lambda_{H} / \mu_{H}\right) \sqrt{2}$.

(Note: this field format, originally conceived by Nambu [39] from quite a different perspective, has been dubbed later as the Higgs field because of its particular property to give mass to spin-1 particles, shown by Higgs [11] and Englert and Brout [12].)

Unfortunately the high non-linearity of this field prevents deriving an analytical solution $\Phi(r)$ from (42) and (38). However, a numerical procedure allows deriving a two-parameter expression for $\Phi(r)$ that closely approximates a true analytical solution. In this approach a generic Ansatz format is adopted for $\Phi(r)$ from which an expression is retrieved of $U(\Phi)$. Subsequently, a fit of is searched on (42). In this approach, first of all, the Euler-Lagrange equation is applied on the static Lagrangian density (38). Hence, from

$$
\frac{\partial \mathrm{L}}{\partial \Phi}-\partial_{i}\left(\frac{\partial \mathrm{L}}{\partial\left(\partial_{i} \Phi\right)}\right)=0
$$

we have from (38),

$\partial_{i} \Phi \partial^{i} \Phi=\frac{d}{d \Phi} U(\Phi)+\rho$

The Ansatz format of the field $\Phi(r)$ is chosen as,

$$
\Phi(r)=\Phi_{0} \frac{\exp (-\lambda r)}{\lambda r}\left\{\frac{\exp (-\lambda r)}{\lambda r}-1\right\}
$$

Substitution of (45) into (43) and subsequent calculation of $U(\Phi)$ gives a fit with (12) for $\mu_{H}^{2}$ and $\lambda_{H}^{2}$, such that

$$
\frac{1}{2} \mu_{H}^{2}=1.06 \lambda^{2} \text { and } \frac{1}{4} \lambda_{H}^{2}=32.3 \frac{\lambda^{2}}{\Phi_{0}^{2}}
$$


The rationale behind this choice (45) is the assumption that the inter-quark potential will behave similarly as the inter-nucleon potential $[40,41]$. The two-parameter field is indistinguishable from the three-parameter field,

$\Phi(r)=\Phi_{0} \exp (-\lambda r)\left\{\frac{1}{(\lambda r)^{2}}-w \frac{1}{\lambda r}\right\}$ for $w=1 / 0.555$

The quark's field would show the characteristics as shown in Figure 3. It would imply that a quark would be repelled by any other quark under influence of the far field, but attracted by the near field, thereby giving rise to mesons as stable two-quark junctions and baryons as three-quark junctions.
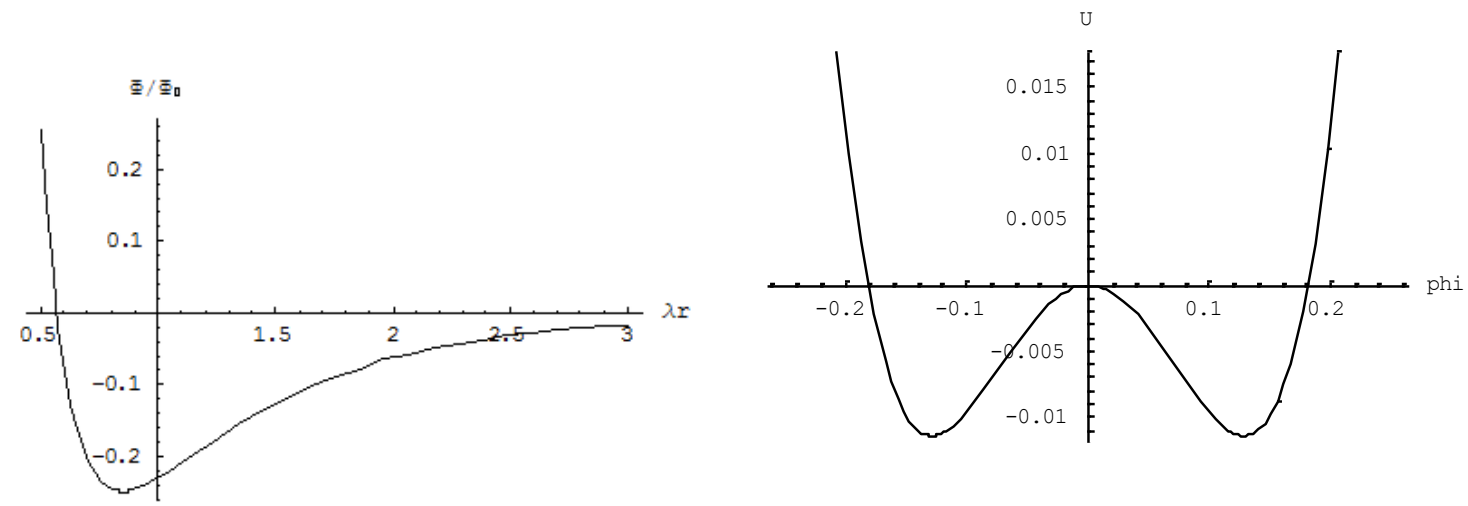

Fig. 3. (Left) The quark's scalar field $\Phi / \Phi_{0}$ as a function of the normalized radius $\lambda x$; (Right) The background field $U_{H}(\Phi)=-U(\Phi)$ retrieved from the spatial expression.

Unfortunately, this radial symmetric format is not viable, because it violates the renormalization constraint. However, comparing (47) with (41) reveals a striking correspondence. Nevertheless, there is a major difference as well. While the derivation of (47) has been based upon a presupposed energetic monopole model for the quark, (41) is the result of a dipole moment next to a monopole. Hence, by restricting the validity of (47) to the dipole axis $x$, the renormalization problem is removed by rewriting (47) as a sum of a far field and a near field, such that,

$\Phi(x)=\Phi_{F}(x)+\Phi_{N}(x)$ with $\Phi_{F}(x)=-w \Phi_{0} \frac{\exp (-\lambda x)}{\lambda x}$ and $\Phi_{N}(x)=\Phi_{0} \frac{\exp (-\lambda x)}{(\lambda x)^{2}}$

Hence, the conclusion is that the field (42), which has the same format as the Higgs field in the Standard Model of particle physics, can be interpreted as the shielded radial symmetric field of an energetic monopole in conjunction with a one-dimensional dipole field. The quark, conceived as a third-type Dirac particle is compatible with this model. The near field is due to the dipole and gives an interpretation for the near field that glues the quarks together in hadronic structures. The shielding of the fields is just the opposite of the antishielding effect that shows up in gravity as a consequence of a background field that consists of virtually mass less elementary dipoles. The break in the field that spoils the symmetry of 
this Debije field, thereby modifying it into the Higgs field, can be ascribed to the quark's polarisable dipole moment.

If it is true that the Higgs field of particle physics can be traced back to a background fluid of polarisable dipoles and the quark's property of possessing a polarisable dipole moment in a classical scalar field evoked by its monopole property, the issue has to be addressed how this field can create massive fermionic and bosonic particles. To address this issue, let us consider the most simple structure of a bond between quarks. A two-quark bond is the most simple one. Because each quark has non-integer spin due to its virtual angular momentum, the bond has integer spin. Hence, the most simple structure is a boson with spin-1, consisting of an elementary quark and an elementary antiquark, thereby composing a pion. Conceiving the pion as a structure in which a quark couples to the field of the antiquark with the generic quantum mechanical coupling factor $g$, the pion can be modeled in its center of mass frame as the one-body equivalent of a two-body oscillator, described by the timeindependent wave equation for its quantum mechanical field $\psi$,

$$
-\frac{\hbar^{2}}{2 m_{m}} \frac{\mathrm{d}^{2} \psi}{\mathrm{d} x^{2}}+\{U(d+x)+U(d-x)\} \psi=E \psi ; \quad U(x)=g \Phi(x),
$$

in which $\Phi(x)$ is the quark's scalar field as derived before and eventually expressed by (48), $2 d$ the quark spacing, $m_{m}$ is the bare mass of the quark (not to be confused with the constituent mass), $V(x)=U(d+x)+U(d-x)$ its potential energy, and $E$ the generic energy constant, which is subject to quantization. Assuming that the bond is stable, the pion gains a ground state energy, which is unrelated to the bare masses of the quark. The equivalent massive energy of this ground state may exceed the sum of massive energy of the bare quark masses. Figure 3 shows a schematic configuration of this bond. It also shows that a quark is much more than just a scalar field. It will be clear from (49) that the potential energy $V(x)$ can be expanded as,

$$
V(x)=U(d+x)+U(d-x)=g \Phi_{0}\left(k_{0}+k_{2} \lambda^{2} x^{2}+\ldots\right),
$$

in which $k_{0}$ and $k_{2}$ are dimensionless coefficients that depend on the spacing $2 d$ between the quarks.

It has to be kept in mind that this model holds in the center of mass frame. Within this frame the quantum mechanical field can be conceived in terms of the non-relativistic Schrödinger one. Nevertheless, a lab frame interpretation of properties derived in this frame will need a relativistic correction because the meson flies at near-light speed. 


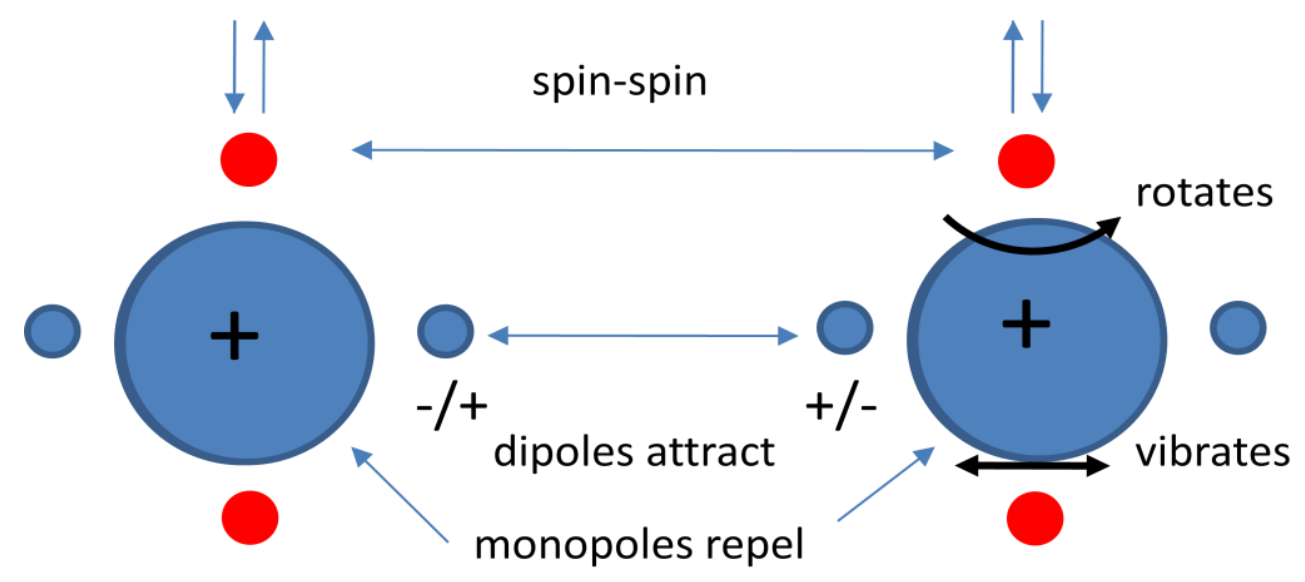

Fig. 3. A quark has two real dipole moments, hence two dipoles. One of these (horizontally visualized) is polarisable in a scalar potential field. The other one (vertically visualized) is not. The dipole moments are subject to spin statistics. However, the polarity of the horizontal one is restrained by the bond: the horizontal dipoles are only oriented in the same direction: either inward to the centre or outward from the centre.

To facilitate the analysis, (49) is normalized as,

$-\alpha_{0} \frac{\mathrm{d}^{2} \psi}{\mathrm{d} x^{\prime 2}}+V^{\prime}\left(x^{\prime}\right)=E^{\prime} \psi$

in which $\alpha_{0}=\frac{\lambda^{2} \hbar^{2}}{2 m_{m} g \Phi_{0}}, x^{\prime}=x \lambda, d^{\prime}=d \lambda, E^{\prime}=\frac{E}{g \Phi_{0}}, U^{\prime}\left(x^{\prime}\right)=\frac{U(\lambda x)}{g \Phi_{0}}$ and

$V^{\prime}\left(x^{\prime}\right)=U^{\prime}\left(d^{\prime}+x^{\prime}\right)+U^{\prime}\left(d^{\prime}-x^{\prime}\right)=k_{0}+k_{2} x^{\prime 2}+\ldots \ldots$

Moreover, from [42, eq. (C2),41],

$\alpha_{0}=\frac{k_{0}^{2}}{2 k_{2}}$

The quantities $\Phi_{0}$ and $\lambda$ are subject to a particular invariant relationship,

$g \frac{\Phi_{0}}{\lambda}=\frac{\alpha \pi \hbar c}{2 d_{\min }^{\prime}}$

in which $g, d_{\min }^{\prime}$ and $\alpha$ are dimensionless constants. Its derivation is beyond the scope of this article. It can be found in previous studies such as [41,43]. The generic quantum mechanical coupling factor $g$ is the square root of the electromagnetic fine structure constant $(g \approx 1 / \sqrt{137})$. The constant $d_{\min }^{\prime}$ is the normalized spacing $d_{\min }^{\prime}=d \lambda$ between the quark and the antiquark in the archetype meson (pion), and $\alpha$ is a numerical factor of order 1. This invariance, dubbed in [44] as the quark scaling theorem, leaves some individual freedom and $\Phi$ and $\lambda$ can therefore be different for different quark flavours. The factor $\alpha$ comes forward from the consideration that the spacing $2 d$ between the quark and the 
antiquark is bridged by a travelling weak interaction boson $\hbar \omega_{W}$, such that the spacing between the two quarks is about a half of its wave length. Hence,

$$
2 d=2 \frac{d_{\min }^{\prime}}{\lambda}=\alpha \frac{c T}{2}=\alpha \frac{c}{2}\left(\frac{2 \pi}{\omega}\right)=\alpha \pi \frac{\hbar c}{\hbar \omega_{W}} \rightarrow \lambda=\frac{2 d_{\min }^{\prime} \hbar \omega_{W}}{\alpha \pi \hbar c}
$$

The archetype, the pion, is the two-quark oscillator in its ground state. The first excitation state transforms a pion into a kaon. The mass ratio between the two is the same as the mass ratio of the normalized energy constants $E^{\prime}-k_{0}$. This is not trivial and it reflects the basic theorem of the theory. This theorem states that the energy wells of the two quarks are not just massive in baryonic sense. Instead, the mass attribute of two-quark junctions (mesons) and three-quark junctions (baryons) is made up by the vibration energy as expressed by the energy state of the quantum mechanical oscillator that they build. The distribution of this mass over constituent quarks is a consequence of this mechanism. Unfortunately, an analytical calculation of the $E^{\prime}-k_{0}$ ratio of kaons over pions, is only possible for the quadratic approximation of the series expansion of the potential energy $V^{\prime}\left(z^{\prime}\right)$. A more accurate calculation requires a numerical approach. A procedure to do so has been documented in [42, Appendix C]. It shows that some simple lines of code in Wolfram's Mathematica [45] may do the job. The numerically calculated ratio of the energy constants appears to be 3.57 instead of 3 as it would have been in the harmonic case. The result explains the excitation of the $137 \mathrm{MeV} / \mathrm{c}^{2}$ pion mass to the $490 \mathrm{MeV} / \mathrm{c}^{2}$ mass of the pseudoscalar kaon. This result gives a first indication for the viability of the quark's potential field as developed. This result also gives rise to the question if other mesons can be regarded as a result from enhanced excitation. Table I gives a survey of the calculated ratios for higher excitation ratios. It gives the pseudoscalar $\eta^{\prime}$ meson as a candidate from second level excitation. The table gives no candidate for third level excitation. As shown in [42], the corresponding level of energy would imply a meson state with a positive value for the binding energy (as is reflected in the value of $k_{0}$ ), which prevents a sustainable quasi-stable configuration. In this calculation, the electromagnetic interactions have been ignored, because their influence is considered to be of second order as compared to the nuclear interaction. Interestingly, the kaon energy does not only correspond with the energy of a pion in its state of first excitation, but also with the ground state energy of a quark junction at smaller spacing, thereby composing the kaon as a $u \bar{s}$ or a $\bar{u} s$ bond, composed by the $u$ quark next to the heavier $s$ quark. Therefore, the excitation mechanism is potentially subject to bootstrapping, showing many excitation and de-excitation routes, resulting in a quasi-chaotic, but nevertheless deterministic, mass spectrum of mesons.

The mechanism as just described allows to calculate the mass spectrum of pseudoscalar mesons. Incorporating the spin-spin interaction between the mesons, as shown in figure 3 allows to include the mass spectrum of vector mesons as well. An extensive review on the mass spectrum of hadrons has been documented in my preprint "On the quark scaling mechanism and the polarisable dipole moment of the quark in a scalar potential field, in which I have described these two unrecognized theoretical elements in more detail [44].

It is also possible to calculate the mass spectrum of baryons as well in a more or less similar way. Whereas a meson can be conceived as the one-body equivalent of a two-body 
harmonic oscillator, a baryon can be conceived as the one-body equivalent of a three-body harmonic oscillator. This is illustrated in Figure 4. The polarisable dipole moment of quarks allows to understand the binding of quarks in a triangular structure from a decomposition of the dipole moments along the baryon axes. This structure, as well as the structure of mesons in pseudoscalar state can be described upon the scalar field composed by the monopole of in conjunction with a dipole of quarks conceived as third-type Dirac particles.

Table I: meson excitations

\begin{tabular}{|l|l|l|l|}
\hline Bottom level & $E_{\text {bind }}^{\prime}=-1 / 2$ & mass ratio & mass in $\mathrm{MeV} / \mathrm{c}^{2}$ \\
\hline Ground state & $E_{0}^{\prime}-E_{\text {bind }}^{\prime}=0.84$ & 1 & $\begin{array}{l}137 \\
\text { (pion = 135-140) }\end{array}$ \\
\hline First excitation & $E_{1}^{\prime}-E_{\text {bind }}^{\prime}=3.00$ & 3.57 & $\begin{array}{l}489 \\
(\text { kaon }=494-498)\end{array}$ \\
\hline Second excitation & $E_{2}^{\prime}-E_{\text {bind }}^{\prime}=6.06$ & 7.21 & $\begin{array}{l}988 \\
\left(\eta^{\prime}=958\right)\end{array}$ \\
\hline Third excitation & $E_{3}^{\prime}-E_{\text {bind }}^{\prime}=9.94$ & 11.83 & $? ? ?$ \\
\hline
\end{tabular}

The classical issue of baryons structures is the problem how three identical quarks may compose a structure in which the quarks are in the same state of energy and spin without violating Pauli's exclusion principle. In a meson structure the problem does not show up, because two mesons may have their nuclear (angular) spins parallel owing to the fact that the quarks show a difference in particle/antiparticle state. The spin conflict in baryons is one of the reasons why in the Standard Model an additional attribute has been assigned to quarks. This attribute is the color charge, which is seen as the origin of gluons that binds the quarks in a baryon together. Since then Quark Color Dynamics (QCD) has become the corner stone of the Standard Model. The classical spin conflict does not necessarily show up in our structural model. The reason is that the polarizable dipole moment represents a quantum mechanical state variable similar to nuclear spin.. Two adjacent quark may switch the state variable without affecting the dipole moment, thereby avoiding the Pauli problem. Hence, this degree of freedom may allow a physical explanation of the color attribute. Although the nuclear spin states of the quarks have no influence on the structural behavior as a multibody (an)harmonic oscillator, they have influence on the state of energy. However, while under influence of excitation or de-excitation (by weak interaction) the flavors of quarks may change, it does not occur under influence of changes in spin (by strong interaction).

The one-body equivalent of the three-body quantum mechanical oscillator can be analyzed in terms of pseudo-spherical Smith Whitten coordinates [46]. The Smith-Whitten system of coordinates is six-dimensional. Next to a (hyper)radius $\rho$, the square of which is the sum of the squared spacings between three bodies, there are five angles $\varphi, \vartheta, \alpha, \beta, \gamma$, in which $\varphi$ and $\vartheta$ model the changes of shape of the triangular structure and in which $\alpha, \beta$ and $\gamma$ are the Euler angles. The latter ones define the orientation of the body plane in 3D-space. The planar forces between three identical interacting bodies are not only the cause of dynamic deformations of the equilateral structure, but are also the cause of a Coriolis effect that result in vibra-rotations around the principal axes of inertia of the three-body structure [47]. 
The application of this approach for baryons has been documented by the author in [48], showing that the wave equation of the quasi-equilateral baryon structure can be formulated as

$-\alpha_{0}\left\{\frac{d^{2} \psi}{d \rho^{\prime 2}}+\frac{5}{\rho^{\prime}} \frac{d \psi}{d \rho^{\prime}}+\frac{R(m, v, k)}{\rho^{\prime 2}} \psi\right\}+V^{\prime}\left(\rho^{\prime}\right)=E^{\prime} \psi$

in which $\alpha_{0}=\frac{\hbar^{2} \lambda^{2}}{6 m g \Phi_{0}} ; E^{\prime}=\frac{E}{3 g \Phi_{0}} ; V^{\prime}=\frac{V}{3 g \Phi_{0}} ; \rho^{\prime}=\rho \lambda$, and

$V\left(\rho^{\prime}\right)=3 g \Phi_{0}\left(k_{0}+k_{2} \rho^{\prime 2}+\ldots \ldots.\right)$

$R(m, v, k)=4 m+|v-k|(4 m+|v-k|+4)$

This wave function is the three-body equivalent of the pion's two-body wave equation shown in (57). In the ground state $m=0$. Hence,

$R=R(0, v, k)=l(l+4) ; l=|v-k|$.

The radial variable $\rho$ is the already mentioned hyper radius. The potential field is just the threefold of the potential field in the wave equation of the pion. There are three quantum numbers involved. Two of those are left in the ground state, effectively bundled to a single one. The quantum number $k$ allows a visual interpretation, while $v$ is difficult to visualize. The impact of $k$ is shown in Figure 4.

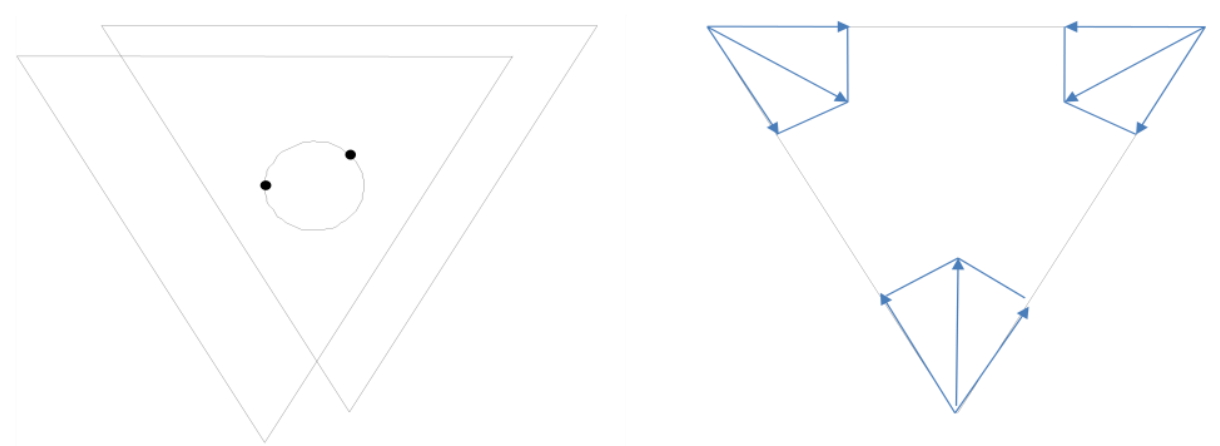

Fig. 4: . Left: physical interpretation of the motion associated with the angular quantum number $k$. Right: decomposition of the dipole moment vectors along the baryon axes.

It illustrates the motion of the center of mass under influence of $k$. Note that this rotation is quite different from a rotation of the triangular frame around the center of mass. It is the center of mass itself that rotates, while the frame does not. Actually, the small motions of the individual quarks are responsible for this motion. As shown in [48], this relatively simple wave function expression allows a pretty accurate calculation of the mass spectrum of baryons. The octet states in the baryon classification are the counter part of the meson 
pseudoscalar states, the decuplet states are the counter part of the vector mesons. A single integer step in the quantum number $l$, brings the $\mathrm{p}, \mathrm{n} / \Delta$ level to the $\Sigma / \Sigma^{*}$-level, etc.

At this point it is instructive relating the structural model as developed so far with the Standard Model. The Standard model has been constructed on the basis of a simple question: how to describe the field of a quark in an ambient field of nuclear forces? The recipe is simple as well: try to find a covariant equivalent for the quark's field in an ambient field from the quark's field in free space. Because the quark has not been recognized as a third type Dirac particle, physical knowledge of the nuclear forces could not been taken into account. Hence, a model for the quark's field has been developed from an abstract point of view. In this view, the nuclear forces are supposed being originating from the axiomatic ambient field, known as the Higgs field, as defined by (42). Rather than being broken by the polarisable dipole moment of the quark, the break is supposed being happened in the early universe due to a Spontaneous Break in a symmetric background field. As a consequence the nuclear forces are described in terms of novel elementary particles, such as the weak interaction $W / Z$ bosons and the strong interaction gluons operating on a family of elementary quark flavors and leptons, eventually adding up to 27 elementary particles under adoption of some additional physical axioms such as isospin and color force. The result is a rigid theoretical framework capable to describe and predict nuclear processes with high precision. That is the good thing. The simple fact, though, is, that the more particles are declared as elementary and the more axioms are added, the more precise a theory will be. The counter side is that quite some particles and physical relationships are declared as elementary and axiomatic while a structural view shows undeniable and predictable relationships between "elementary" particles. In my preprint "On the quark scaling theorem and the polarisable dipole of the quark in a scalar field" [44], these relationships are discussed in depth. Within the scope of this article, which is aimed to show a relationship between the cosmological background field and the Higgs field, it will not further be discussed.

To obtain our goal, somewhat more profiling of a quark will be required. As concluded so far, the potential field of the quark is composed by the sum of a far field from the monopole and a near field from the polarisable dipole moment. This dipole moment $m_{p} d=\hbar / 2 c$, in which $m_{p}$ and $d$ are unknown quantities, creates a near field potential field $\Phi_{G N}(x)$ along the dipole axis $x$ such that,

$\Phi_{G N}(x)=\frac{G m_{p} d}{x^{2}} \rightarrow \Phi_{G N}(x)=\frac{\hbar}{2 c} \frac{\lambda_{0}^{2} G}{\left(\lambda_{0} x\right)^{2}} \rightarrow \Phi_{G N}(x)=\Phi_{0}^{\prime} \frac{1}{\left(\lambda_{0} x\right)^{2}} ; \Phi_{0}^{\prime}=\frac{\hbar}{2 c} G \lambda_{0}^{2}$.

(the double accent in $\Phi_{0}^{\prime}$ is meant for distinguishing it from $\Phi_{0}$ later)

Note that there is no particular reason yet to identify the $\lambda_{0}$ in (57) as the $\Lambda$-related one in $(11,12)$. Apart from the near field $\Phi_{G N}(x)$, the quark spreads a far field $\Phi_{G F}(r)$. The far field is the result of the (monopole) mass $m_{q u}\left(\neq m_{p}\right.$ ) of a bare quark. Under absence of any mass generation mechanism, only the eigen value $\hbar / 2$ of the quark's elementary angular momentum is left as the manifestation of this bare mass (as discussed and proven in [44], 
the bare mass is different from the quark's constituting mass resulting from the mass generation mechanism). Interpreting the angular momentum as a virtual rotation with light speed at a fictitious radius $r_{0}=1 / w \lambda_{0}$, in which $w$ is an unknown dimensionless weighting constant, we have

$$
\frac{\hbar}{2}=\frac{m_{q u} c}{w \lambda_{0}} \rightarrow m_{q u}=w^{\prime} \frac{\hbar}{2} \frac{\lambda_{0}}{c}
$$

Hence, from classical field theory,

$$
\Phi_{G F}(r)=\frac{m_{q u} G}{r}=w^{\prime} \frac{\hbar}{2} \frac{\lambda_{0}}{c} \frac{G}{r}=w^{\prime} \frac{\hbar}{2} \frac{G \lambda_{0}^{2}}{c} \frac{1}{\lambda_{0} r}
$$

and, under consideration of $\Phi_{0}$ as defined in (57),

$$
\Phi_{G F}(r)=w^{\prime} \frac{\hbar}{2} \frac{G \lambda_{0}^{2}}{c} \frac{1}{\lambda_{0} r}=\Phi_{0}^{\prime} \frac{w^{\prime}}{\left(\lambda_{0} r\right)}
$$

Hence, the potential field of the quark along the axis set up between the dipole axis can be expressed as an energy $\Phi(\lambda x)$ such that

$$
\Phi(\lambda x)=\Phi_{0}^{\prime}\left\{\frac{1}{\left(\lambda_{0} x\right)^{2}}-w^{\prime} \frac{1}{\lambda_{0} x}\right\} ; \quad \Phi_{0}^{\prime}=m_{q u} \frac{\hbar}{2 c} G \lambda_{0}^{2}
$$

Similarly as the field of darks, the quark field is influenced by the energetic background field. However, where the gravitational field is enhanced under polarization of the background particles, the nuclear field is suppressed. This can be understood by re-inspection of (11). There is no reason why space-time curving would be restricted to massive energy. It may occur under a more general interpretation of energy as well. Moreover, the curving might be negative instead of positive like assumed for gravity. Hence, let us rewrite (11) as,

$$
-\frac{\partial^{2}}{c^{2} \partial t^{2}}(r \Phi)+\frac{\partial^{2}}{\partial r^{2}}(r \Phi)-\lambda^{2}(r \Phi)=-r \frac{8 \pi \Phi_{0}}{c^{2}} \delta^{3}(r) U(t),
$$

Like noted before, its solution under static conditions is given by (15). By taking the influence of $\lambda^{2}$ in account, (61) is modified into

$$
\Phi(\lambda x)=\Phi_{0}^{\prime} \exp (-\lambda x)\left\{\frac{1}{\left(\lambda_{0} x\right)^{2}}-w^{\prime} \frac{1}{\lambda_{0} x}\right\} ; \quad \Phi_{0}^{\prime}=m_{q u} \frac{\hbar}{2 c} G \lambda_{0}^{2},
$$

in which $m_{q u}$ is the unknown mass of the bare quark and in which $\lambda$ is identified as the unknown decay parameter of the field due to the background energy. Note that unlike $\lambda_{0}$, 
this $\lambda$ has the same semantics now as in the gravity case, i.e., related with Einstein's $\Lambda$ as $\lambda^{2}=2 \Lambda$. Rewriting (63) in terms of $\lambda x$ gives,

$\Phi(\lambda x)=\Phi_{0}^{\prime} \exp (-\lambda x)\left\{\left(\frac{\lambda}{\lambda_{0}}\right)^{2} \frac{1}{(\lambda x)^{2}}-\left(\frac{\lambda}{\lambda_{0}}\right) w^{\prime} \frac{1}{\lambda x}\right\}=\Phi_{0}^{\prime}\left(\frac{\lambda}{\lambda_{0}}\right)^{2} \exp (-\lambda x)\left\{\frac{1}{(\lambda x)^{2}}-w \frac{1}{\lambda x}\right\}$,

in which $w=w^{\prime} \frac{\lambda_{0}}{\lambda}$. Defining

$\Phi_{0}=\Phi_{0}^{\prime}\left(\frac{\lambda}{\lambda_{0}}\right)^{2}=m_{q u} \frac{\hbar}{2 c} G \lambda_{0}^{2}\left(\frac{\lambda}{\lambda_{0}}\right)^{2}=m_{q u} \frac{\hbar}{2 c} G \lambda^{2}$

Eq.(63) is rewritten as,

$\Phi(\lambda x)=\Phi_{0} \exp (-\lambda x)\left\{\frac{1}{(\lambda x)^{2}}-w \frac{1}{\lambda x}\right\} ; \Phi_{0}=m_{q u} \frac{\hbar}{2 c} G \lambda^{2}$

Note that $m_{q u}$ still is the mass of the bare quark. We are back now on eq. (41) with the exception that a profile has been given to the bare mass of a quark.

From (58) and from the relation between $w$ and $w^{\prime}$ as just given, we have, under consideration of (47),

$m_{q u}=w \frac{\hbar}{2 c} \lambda_{0} ; w \approx 1 / 0.55$

A more extensive review on the nuclear background energy can be found in the preprint "On the quark scaling theorem and the polarisable dipole of the quark in a scalar field" [44]. The summary given in this paragraph illustrates that, under recognition of the quark's identity as a particular Dirac particle that possesses a polarisable dipole moment in a scalar field, it makes sense to analyze its field prior to QFT's second quantization. Doing so, the mass spectrum of hadrons can be calculated in a deterministic way as well as the constituent mass relations between the quark flavors, thereby revealing that baryonic mass is the result of the ground state energy of quantum mechanical oscillators built by interacting quarks.

\section{Relating cosmological properties with nuclear properties}

From the analyses made so far, it is fair to conclude that quarks in a meson and the cosmological darks are Dirac particles. In that sense they are similar to electrons. However, whereas the field of an electron is not affected in vacuum, the field of a quark is shielded by an energetic background field while the field of a dark is enhanced by such a field. To enable a proper comparison between the three particle types, a generic force $F$ will be defined as the spatial derivative of a generic potential $\Phi$ in units of energy, such that for electrons, darks and quarks, respectively, 


$$
\begin{aligned}
& F=\frac{\partial}{\partial y} \Phi=e \frac{\partial}{\partial y} \Phi_{\mathrm{e}} \\
& F=\frac{\partial}{\partial y} \Phi=m_{0} \frac{\partial}{\partial y} \Phi_{\mathrm{G}} \\
& F=\frac{\partial}{\partial y} \Phi=g \frac{\partial}{\partial y} \Phi_{\mathrm{qu}},
\end{aligned}
$$

In which $q, m_{0}$ and $g$, are the coupling factors of, respectively, an electron, a classical massive particle and a quark to respectively, an electric potential $\Phi_{\mathrm{e}}$, a gravitational potential $\Phi_{\mathrm{G}}$ and a nuclear potential $\Phi_{\mathrm{qu}}$. Electroweak unification relates the nuclear coupling factor $g$ with the electromagnetic coupling factor $e$ by the fine structure relationship $e^{2}=4 \pi \varepsilon_{0} \hbar c g^{2}$. Whereas these potential fields are specific and have specific dimensionalities, they are all derived from a generic potential $\Phi$ in the dimension of energy.

As discussed in paragraph 3, a classical (baryonic) mass $m_{0}$ feels a gravitational force from another classical mass $m_{0}$ as,

$$
F=m_{0} \frac{\partial}{\partial x} \Phi_{\mathrm{G}} ; \Phi_{G}=-m_{0} G \lambda \frac{\cos \lambda x+\sin \lambda x}{\lambda x}
$$

The potential field of the quark modeled has been found in (64) as, as

$$
\Phi(\lambda x)=\Phi_{0} \exp (-\lambda x)\left\{\frac{1}{(\lambda x)^{2}}-w \frac{1}{\lambda x}\right\} ; \quad \Phi_{0}=m_{q u} \frac{\hbar}{2 c} G \lambda^{2}
$$

According to the theory developed in the previous chapters, a quark feels a force from another quark as,

$$
F=g \frac{\partial}{\partial r} \Phi_{\text {qu }}
$$

The format of the field $\Phi_{\mathrm{qu}}$ matches with the format of the field (68). Recognizing the origin of the far field component of the nuclear force as a Newtonian field, we have

$$
F=g \frac{\partial}{\partial r} \Phi=\beta m_{q u} \frac{\partial}{\partial r} \Phi_{\mathrm{G}}
$$

in which $\beta$ is a dimensionless unknown proportionality factor. Hence, from (68) and(70),

$$
g\left(m_{q u} \frac{\hbar}{2} \frac{G \lambda^{2}}{c}\right)=\beta m_{q u}^{2} G \lambda \rightarrow m_{q u}=\frac{g}{\beta} \frac{\hbar}{2 c} \lambda
$$


Note the difference with (65). It implies that the bare mass $m_{q u}$ of the quark depends on an elementary dipole moment with eigen value $\hbar / 2 c$ with dimensionality [mass $\mathrm{x}$ length] multiplied by $\lambda$ with dimensionality [length ${ }^{-1}$ ]. This mass quantity seems identifying the weak interaction between quarks as the equivalent of a Newtonian gravitational interaction as if a quark were an ordinary massive particle in classical gravitational sense. However, if the quark would have the same gravitational properties as a baryonic pointlike mass, the shielding of its potential field by the energetic background field would be the same. In fact, relationship (70) reveals that whereas two baryonic masses attract under Newton's law, two quark bare masses repel under Newton's law. It also means that whereas the gravitational dipoles in the energetic background field enhance the potential field of a baryonic source, modeled as a pointlike mass, the same dipoles shield the nuclear potential field of the quark.

Recognizing the role of $\lambda$ in gravity in terms of Einstein's $\lambda_{M}^{2}=2 \Lambda$, we have from (14), under the recognition that the quark's bare mass $m_{q u}$ is a Newtonian anti-gravitational particle,

$M \lambda_{M}^{2}=m_{q u} \lambda^{2}=\frac{2}{5} \frac{a_{0}}{G}$

This is a basic formula for the unification of gravity with quantum physics. From (71) and (72),

$\frac{a_{0}}{G}=\frac{5}{2} \frac{g}{\beta} \frac{\hbar}{2 c} \lambda^{3}$

The remaining issue for relating the relationship between cosmological quantities with nuclear quantities is establishing a value for the quark's quantity $\lambda$. It has been shown before in (54) that in the center of mass of a pion,

$\lambda=\frac{2 \hbar \omega_{W} d_{\min }^{\prime}}{\alpha \pi \hbar c}$

Because this relation holds in the center of mass frame of a pion, a relativistic correction is needed from $\lambda \rightarrow \lambda_{00}$. Considering that the energy of the pion is dominated by the binding energy between the quark and the antiquark in a meson, which is provided by the weak interaction boson, we may relate the massive energy $\hbar \omega_{\pi}$ of a pion if it would be in rest with the massive energy $\hbar \omega_{W}$ of the weak interaction boson as,

$$
\frac{\hbar \omega_{W}}{\hbar \omega_{\pi}}=\frac{\lambda}{\lambda_{00}}
$$

Hence, from (72-75), 


$$
\frac{a_{0}}{G}=\frac{5}{2} \frac{g}{\beta} \frac{\hbar}{2 c} \lambda_{00}^{3}=\frac{5}{2} \frac{g}{\beta} \frac{\hbar}{2 c}\left(\frac{2 \hbar \omega_{\pi} d_{\min }^{\prime}}{\alpha \pi \hbar c}\right)^{3}
$$

or, equivalently, with $\gamma=\alpha \sqrt[3]{\beta}$,

$$
\frac{a_{0}}{G}=\frac{5}{2} g \frac{\hbar}{2 c}\left(\frac{2 \hbar \omega_{\pi} d_{\min }^{\prime}}{\gamma \pi \hbar c}\right)^{3}
$$

With $G=6.6710^{-11} \mathrm{~kg}^{-1} \mathrm{~m}^{3} \mathrm{~s}^{-2}, \hbar \omega_{\pi} \approx 140 \mathrm{MeV}$ (pion) and $g=1 / \sqrt{137}$, the dimensionless correction factor should amount to $\gamma \approx 1.05$ for giving a fit with Milgrom's acceleration constant $a_{0} \approx 1.2510^{-10} \mathrm{~m} / \mathrm{s}^{2}$.

It is rather satisfying, but in fact surprising, that the fit factor $\gamma$ is close to unity. Unfortunately it is just a fit and not more than that. Let us consider how it has been built up. Different from the contribution $\beta$, the contribution from $\alpha$ can be scientifically numerically explained. Like mentioned before, the factor $\alpha$ comes forward from the consideration that the spacing $2 d$ between the quark and the antiquark is bridged by a travelling weak interaction boson $\hbar \omega_{W}$, such that the spacing between the two quarks is about a half of its wave length, such as expressed by (74). Hence, it is tempting to expect that $\alpha$ would be equal to one. Actually, it is slightly smaller because of the following reason. Let us inspect eqs. (62). This equation represents the wave equation of the far field boson. It has the format of Proca's equation for a massive photon. With the interpretation discussed in this article, it is the wave equation for a mass less boson hampered by the background field. The associated boson has an energy equal to $\lambda(\hbar c)$. Experimentally, though, the boson will show up as a twin pair as a consequence of the break between two quarks in high energy particle collisions. In the decay paths of this bosonic energy, the energy is not distinguishable from the energy of a single particle, which has been dubbed as the Higgs boson with energy,

$$
m_{H}^{\prime}=2 \lambda(\hbar c)
$$

Hence, from (74) and (78),

$$
\alpha=\frac{4 d_{\min }^{\prime}}{\pi} \frac{\hbar \omega_{W}}{m_{H}^{\prime}} \approx 0.69 \text { for } \hbar \omega_{W}=80.4 \mathrm{GeV} \text { and } m_{H}^{\prime}=126 \mathrm{GeV}
$$

This view can be supported by two additional arguments. The first is that from canonical theory $m_{H}^{\prime} / \hbar c=\mu_{H} \sqrt{2},[1]$, which under consideration of (46) gives $m_{H}^{\prime} \approx 2 \lambda(\hbar c)$. The second argument is that $\alpha \approx 0.69$ is just the same value as shows up in the calculation of the gravitation constant $G$ in terms of quantum mechanical parameters, reported back in 2016, [43], and before in 2011, [41]. Under consideration of this result, $\beta \approx 3.52$. From (71) we have for the bare mass of the quark,

$$
m_{q u}=\frac{g}{\beta} \frac{\hbar}{2 c} \lambda=\frac{g}{\beta} \frac{\hbar}{4 c^{2}} 2 \lambda c=\frac{g}{\beta} \frac{m_{H}^{\prime}}{4 c^{2}} \rightarrow m_{q u}^{\prime}=\frac{g}{4 \beta} m_{H}^{\prime}
$$


which after correction to the lab frame value, reduces to,

$m_{q u}^{\prime}=\frac{g}{4 \beta} m_{H}^{\prime}\left(\frac{\hbar \omega_{\pi}}{\hbar \omega_{W}}\right) \approx 1.32 \mathrm{MeV}$

Unfortunately, no experimental evidence is available for the quark's bare mass. Anyhow, its order of magnitude corresponds with the one estimated by the Particle Data Group (PDG), [49].

\section{Discussion and conclusion}

Expression (77) relates the gravitational constant $G$ and Milgrom's acceleration constant $a_{0}$ as the major gravitational parameters with a single nuclear parameter (the pion's rest mass). It will be clear that this result reveals an extremely simple relationship between gravity and quantum physics. It is obtained by a theory that solves the so called "Cosmological Constant catastrophe" as well. This catastrophe is the disagreement between the observed values of vacuum energy density and the theoretical enormously large value of zero-point energy of the quantum field theory. The problem vanishes in the theory developed in this article, in which the cosmological background energy and the nuclear background energy have appeared being the same as being captured in elementary polarisable energetic particle with a volume density shown by (27) as,

$$
N / \mathrm{m}^{3}=\frac{a_{0}}{20 \pi G} \frac{2 c}{\hbar}
$$

giving an amount of $1.7 \times 10^{14}$ particles per cubic nanometer.

This equivalence is due to the reason that both gravity and quantum physics have been described as the modulation of space-time curving on top of a bias in a weak-field approximation of Einstein's Field Equation (effectively, removing the bias is not different from renormalization). In this view the bias is considered as an irrelevancy that can be ignored. Whereas in gravity the bias is usually ignored indeed, it is not the case in canonical particle quantum physics.

\section{Summarizing:}

In this article, quarks, as the elementary nuclear particles, and darks as elementary constituents of the cosmological background energy, have been described as Dirac particles of a particular kind, dubbed as thirds, being subject to a classical potential field. This has been possible by recognizing that these particular Dirac particles show a real valued polarisable second elementary dipole moment next to the well known elementary angular moment. Quite surprisingly, while the dark behaves as a common gravitational Newtonian particle, the bare quark behaves as an anti-gravitational Newtonian particle (while the constituent mass of a quark is gravitational, the bare mass is anti-gravitational). As a result of this difference, the potential field of quarks is shielded by the darks, while the 
gravitational field of a baryonic kernel is enhanced by the darks, thereby giving an explanation for the dark matter effect in cosmology. It has been shown that the theory developed in this article has resulted in the view that gravity and quantum physics can be unified. The viability of the theory is proven by two verifiable relationships. The first one, documented by me before [9], is the calculated value of Milgrom's acceleration constant $a_{0}$ from the baryonic content $\Omega_{B}(=0.0486)$ of the universe, shown in (14) as,

$a_{0}=\frac{15}{4} \Omega_{B} \frac{c}{t_{H}}$

in which Hubble time $t_{H} \approx 13.8$ Gyear, which gives $a_{0}=1.2510^{-10} \mathrm{~m} / \mathrm{s}^{2}$. The second one is the novel relationship, shown by (77), between Milgrom's acceleration constant as characteristic cosmological quantity with the energetic equivalent $\hbar \omega_{\pi}$ of the pion mass as the characteristic nuclear quantity, with constants of nature, $G, \hbar, c$ and $g\left(g^{2}=1 / 137\right)$. From the fit between (83) and (77) it seems fair to conclude that the nuclear background field known as the Higgs field and the cosmological background field assigned to the Cosmological Constant are identical and is embodied by the darks with a particle density shown in (82).

\section{APPENDIX: THE GRAVITATIONAL WAVE EQUATION}

The main reason of including the appendix is to show the validity range for the weak field limited modification of Newton's gravitation law, due to Einstein's gauge constant $\Lambda$. To do so properly, the derivation requires a short summary of common textbook stuff without $\Lambda$, before extending it to meet the objective. This objective implies that we have to solve Einstein's Field Equation for a spherically symmetric space-time metric that is given by the line element (19),

$$
\mathrm{d} s^{2}=g_{t t}(r, t) \mathrm{d} q_{0}^{2}+g_{r r}(r, t) \mathrm{d} r^{2}+r^{2} \sin ^{2} \vartheta \mathrm{d} \varphi^{2}+r^{2} \mathrm{~d} \vartheta^{2}
$$

where $q_{0}=\mathrm{i} c t$.

Note: The space-time (ict, $r, \vartheta, \varphi$ ) is described on the basis of the "Hawking" metric $(+,+,+,+)$. Once more, I would like to emphasize its merit that, by handling time as an imaginary quantity instead of a real one, the ugly minus sign in the metric $(-,+,+,+)$ disappears owing to the obtained full symmetry between the temporal domain and the spatial one.

The components $g_{\mu \mu}$ compose the metric tensor $g_{\mu \nu}$, which determine the Ricci tensor $R_{\mu \nu}$ and the Ricci scalar $R$. These quantities play a decisive role in Einstein's Field Equation, which reads as

$G_{\mu v}+\Lambda g_{\mu v}=\frac{8 \pi G}{c^{4}} T_{\mu v}$ with $G_{\mu v}=R_{\mu \nu}-\frac{1}{2} R g_{\mu \nu}$ 
In a space without massive sources, the Einstein Field Equation under this symmetric spherical isotropy, reduces to a simple set of equations for the elements $R_{\mu \mu}$ of the Ricci tensor,

$$
\begin{array}{ll}
R_{t t}-\frac{1}{2} R g_{t t}+\Lambda g_{t t}=0 ; & R_{r r}-\frac{1}{2} R g_{r r}+\Lambda g_{r r}=0 ; \\
R_{\vartheta \vartheta}-\frac{1}{2} R g_{\vartheta \vartheta}+\Lambda g_{\vartheta \vartheta}=0 ; & R_{\varphi \varphi}-\frac{1}{2} R g_{\varphi \varphi}+\Lambda g_{\varphi \varphi}=0 .
\end{array}
$$

Let us proceed by considering the Ricci scalar. It is defined generically as

$$
R=\sum_{\mu=0}^{3} \sum_{v=0}^{3} g^{\mu v} R_{\mu v}
$$

In spherical symmetry the matrices contain diagonal elements only, so that $(A-4)$ reduces to

$$
R=\sum_{\mu=0}^{3} g^{\mu \mu} R_{\mu \mu} .
$$

This result can be applied to (A-3). Multiplying the first one with $g^{00}\left(=g^{t t}\right)$, the second one with $g^{11}$, etc., and subsequent addition results of the terms $\mu=1,2,3$ gives,

$\sum_{\mu=1}^{3} g^{\mu \mu} R_{\mu \mu}-\frac{3}{2} R+3 \Lambda=-g^{t t} R_{t t}+\sum_{\mu=0}^{3} g^{\mu \mu} R_{\mu \mu}-\frac{3}{2} R+3 \Lambda=-g^{t t} R_{t t}-\frac{1}{2} R+3 \Lambda=0$,

hence $g^{t t} R_{t t}=-\frac{1}{2} R+3 \Lambda$.

Repeating this recipe for $g_{\mu \mu}\left(=1 / g^{\mu \mu}\right)$, we have for reasons of symmetry

$$
g^{\mu \mu} R_{\mu \mu}=-\frac{1}{2} R+3 \Lambda
$$

Note that the subscripts and superscripts $00,11,22$, and 33 are, respectively, identical to $t t, r r, \vartheta \vartheta$ and $\varphi \varphi$. Applying this result to Einstein's equation set gives,

$$
2 g^{\mu \mu} R_{\mu \mu}-2 \Lambda=\frac{8 \pi G T_{\mu \mu} g^{\mu \mu}}{c^{4}}
$$

such that after multiplication by $g_{\mu \mu}$, we have

$$
2 R_{\mu \mu}-2 g_{\mu \mu} \Lambda=\frac{8 \pi G T_{\mu \mu}}{c^{4}}
$$


Let us first proceed under the conditions of the absence of massive sources ( $\left.T_{\mu \mu}=0\right)$ and let us consider the Ricci tensor components $R_{t t}$ and $R_{r r}$ under use of the results shown in Table A-1, that can be found in basic textbooks [31]. Note: $g^{\prime}$ and $g^{\prime \prime}$ means differentiation, respectively double differentiation of $g$ into $r ; \dot{g}$ and $\ddot{g}$ means differentiation, respectively double differentiation of $g$ into $t$. Multiplying (A-3a) by $1 / g_{t t}$ and (A-3b) by $1 / g_{r r}$ gives,

$\frac{R_{t t}}{g_{t t}}-\Lambda=0$ and $\frac{R_{r r}}{g_{r r}}-\Lambda=0$

which, under the assumption of a zero Cosmological Constant $(\Lambda=0)$, after subtraction and under use of the expressions in Table A-1 results into.

$-\frac{1}{r} \frac{1}{g_{r r}}\left(\frac{g_{r r}^{\prime}}{g_{r r}}+\frac{g_{t t}^{\prime}}{g_{t t}}\right)=0$

Hence

$$
\frac{g_{r r}^{\prime}}{g_{r r}}+\frac{g_{t t}^{\prime}}{g_{t t}}=0
$$

which can be integrated to (the Schwarzschild condition),

$$
g_{r r} g_{t t}=1
$$

This, in turn, gives

$$
\frac{\dot{g}_{r r}}{g_{r r}}+\frac{\dot{g}_{t t}}{g_{t t}}=0
$$

Using (A-13), (A-15) and the Table A-1 values on $R_{t t}$ gives

$$
R_{t t}=\frac{1}{g_{r r}}\left(-\frac{1}{2} g_{t t}^{\prime \prime}-\frac{1}{r} g_{t t}^{\prime}-\frac{\ddot{g}_{r r}}{2 c^{2}}\right)=\frac{1}{g_{r r}}\left(-\frac{1}{2 r} \frac{\partial^{2}\left(r g_{t t}\right)}{\partial r^{2}}+\frac{1}{2 c^{2}} \frac{\partial^{2} g_{t t}}{\partial t^{2}}\right) .
$$

Hence, from (A-10) and (A-16),

$$
\frac{2}{g_{r r}}\left(-\frac{1}{2 r} \frac{\partial^{2}\left(r g_{t t}\right)}{\partial r^{2}}+\frac{1}{2 c^{2}} \frac{\partial^{2} g_{t t}}{\partial t^{2}}\right)=\frac{8 \pi G T_{t t}}{c^{4}}
$$

or, equivalently,

$$
-\frac{1}{r} \frac{\partial^{2}\left(r g_{t t}\right)}{\partial r^{2}}+\frac{1}{c^{2}} \frac{\partial^{2} g_{t t}}{\partial t^{2}}=\frac{8 \pi G T_{t t}}{c^{4}} g_{r r}
$$


Applying well-known conditions,

$\Lambda=0$ (already assumed) (no cosmological constant)

$g_{t t}(r, t)=1+h_{\varphi}(r, t)$, where $\left|h_{\varphi}(r, t)\right|<<1 \quad$ (the weak field limit)

$T_{t t}=M c^{2} \delta^{3}(r), \quad$ (pointlike massive source)

$(A-19 a, b, c)$

yields the proper wave equation

$\frac{\partial^{2}\left(r h_{\varphi}\right)}{\partial r^{2}}-\frac{1}{c^{2}} \frac{\partial^{2} r h_{\varphi}}{\partial t^{2}}=-\frac{8 \pi G M}{c^{2}} r \delta^{3}(r) U(t)$,

where $U(t)$ is Heaviside's step function. In the static regime, the equation results into

$$
\frac{1}{r} \frac{\partial^{2}\left(r h_{\varphi}\right)}{\partial r^{2}}=-\frac{8 \pi G M}{c^{2}} \delta^{3}(r) .
$$

This is similar to Poisson's equation,

$$
\nabla^{2} \Phi=\frac{1}{r} \frac{\partial^{2}(r \Phi)}{\partial r^{2}}=-4 \pi G \rho=-4 \pi G M \delta^{3}(r)
$$

the solution of which is the Newtonian potential,

$$
\Phi=-\frac{G M}{r}\left[\mathrm{~m}^{2} \mathrm{~s}^{-2}\right]
$$

Comparing (A-20) with (A-22) gives the equivalence

$$
h_{\varphi}=\frac{2 \Phi}{c^{2}}
$$

So far, this is just textbook stuff, such as can be found, for example, in [31]. It is needed as a basis for deriving the conditions under which the modification of the $\Lambda=0$ wave equation (A-20) toward the $\Lambda \neq 0$ one shown in (28) is justified. Let us first consider the case $\Lambda \neq 0$ under absence of a massive source. Obviously, (A-10) is only satisfied if the influence of the cosmological constant is counter balanced by the hypothetical source

$$
T_{t t}=-p \Lambda, \text { where } p=\frac{c^{4}}{8 \pi G}
$$

Because all four members of the Einstein set $(A-10)$ have to be satisfied, we have, under consideration of (A-10) and Table A1, 
$T_{\mu \mu}=-p g_{\mu \mu} \Lambda$ and $g_{\mu \mu}=\left(1,1, r^{2} \sin ^{2} \vartheta, r^{2}\right)$

This particular stress-energy tensor with equal diagonal elements corresponds with the one for a perfect fluid in thermodynamic equilibrium. So, where empty space corresponds with virtual sources $T_{\mu \mu}=0$, the fluidal space corresponds with virtual sources $T_{\mu \mu}=-p \Lambda$, with $g_{\mu \mu}=\left(1,1, r^{2} \sin ^{2} \vartheta, r^{2}\right)$. Insertion of a massive pointlike source in this fluid and modifying (A-17) by adding the virtual sources, after redefining the weak limit condition as,

$g_{t t}(r, t)=1+h_{t \varphi}(r, t) ; g_{t r}(r, t)=1+h_{r \varphi}(r, t) ;\left|h_{t \varphi}\right|,\left|h_{r \varphi}\right|<<1$,

gives, for the static parts,

$$
\begin{aligned}
& \left.\frac{2}{g_{r r}}\left\{-\frac{1}{2} h_{t \varphi}^{\prime \prime}+\frac{h_{t \varphi}^{\prime}}{4}\left(h_{r \varphi}^{\prime}+h_{t \varphi}^{\prime}\right)-\frac{1}{r} h_{t \varphi}^{\prime}\right)\right\}-2 \Lambda g_{t t}=\frac{8 \pi G T_{t t}}{c^{4}}-2 \Lambda \rightarrow \\
& -h_{t \varphi}^{\prime \prime}-2 \Lambda h_{t \varphi}+\frac{h_{t \varphi}^{\prime}}{2}\left(h_{r \varphi}^{\prime}+h_{t \varphi}^{\prime}\right)-\frac{2}{r} h_{t \varphi}^{\prime}=\frac{8 \pi G T_{t t}}{c^{4}} g_{r r}
\end{aligned}
$$

and, secondly,

$$
-h_{t \varphi}^{\prime \prime}-2 \Lambda h_{r \varphi}+\frac{h_{t \varphi}^{\prime}}{2}\left(h_{r \varphi}^{\prime}+h_{t \varphi}^{\prime}\right)+\frac{2}{r} h_{r \varphi}^{\prime}=0
$$

As long as $\Lambda=0$, and assuming a pointlike source embodied in $T_{t t}$, the Schwarzschild condition shows up. This is obvious by subtracting the latter equation from the former one, thereby allowing exclusion of a singularity at $r=0$. It reveals that, under this condition, the homogeneous formats of the two equations are identical. However, because this is no longer true for $\Lambda \neq 0$, we have to cope with two equations. These two equations are non-linear. However, because $h_{t \varphi}$ and $h_{r \varphi}$ are small in the weak field limit, the two equations can be linearized under the condition that the last term in the left-hand part of these equations is dominant over their preceding terms. This assumption, to be checked later, allows to rewrite (A-26) for $r \neq 0$, as

$$
\left(r h_{t \varphi}\right)^{\prime \prime}+2 \Lambda\left(r h_{t \varphi}\right)=0
$$

A simple format for the second equation is obtained after subtraction (A-27) from (A-26), resulting into,

$$
-2 h_{r \varphi}^{\prime}+2 \Lambda r h_{r \varphi}=2 h_{t \varphi}^{\prime}+2 \Lambda r h_{t \varphi} .
$$

Obviously, $h_{r \varphi}$ can be calculated as soon as $h_{t \varphi}$ is found as a solution of (A-28). Re-inserting the pointlike source, similarly as in the case $\Lambda=0$ and including the time derivatives, yield a wave equation as a generalization of (A-28). After rewriting, 
$h_{t \varphi}=\frac{2 \Phi}{c^{2}}$ and $\Lambda=\frac{\lambda^{2}}{2}$

we have from $(\mathrm{A}-28)$ the inhomogeneous generalization,

$$
-\frac{\partial^{2}}{c^{2} \partial t^{2}}(r \Phi)+\frac{\partial^{2}}{\partial r^{2}}(r \Phi)+\lambda^{2}(r \Phi)=-r \frac{8 \pi G M}{c^{2}} \delta^{3}(r) U(t)
$$

If $\Lambda<0$, we have under static conditions, a similarity with Helmholtz' equation [54] with the screened Poisson's equation, the solution of which is Yukawa's potential,

$\Phi=\frac{G M}{r} \exp (-\lambda r)$

which reduces to Poisson's one for $\lambda \rightarrow 0$.

If $\Lambda>0$, we have under static conditions, a similarity with Helmholtz' equation with a characteristic solution,

$\Phi=\frac{G M}{r}\{\cos \lambda r+\sin \lambda r\}$

This solution reduces to Poisson's one for $\lambda \rightarrow 0$ as well.

This is the weak field limit solution of Einstein's Equation if one does not take the validity of Poisson's equation of gravity for granted, but adopts Helmholtz equation instead under an appropriate choice of the Cosmological Constant.

We are not done yet. There are two remaining issues. The first one is the justification of the linearization approximation by moving from (A-26) to (A-28). Moreover, we have to take into consideration that, although the derived gravitational potential field satisfies (A-3a) and (A$3 b)$, we are not sure that it satisfies $(A-3 c)$ and $(A-3 d)$ as well. It should do, to prevent violation of the metric (A-1). Assessment of it is the second thing to be done.

The remaining issues: (a) the linearization approximation

The linearity approximation $(A-26) \rightarrow(A-28)$ is justified as long as

$$
\left|\frac{2}{r} h_{t \varphi}^{\prime}\right| \gg>\left|\frac{h_{t \varphi}^{\prime}}{2}\left(h_{r \varphi}^{\prime}+h_{t \varphi}^{\prime}\right)\right| \rightarrow \frac{1}{r}>>\frac{1}{4}\left|h_{r \varphi}^{\prime}+h_{t \varphi}^{\prime}\right| .
$$

Under consideration of (A-29), it can be written as,

$\frac{1}{\Lambda r^{2}}>>\frac{1}{4}\left|h_{r \varphi}-h_{t \varphi}\right|$

This condition enforces calculation of $h_{r \varphi}$ from $h_{t \varphi}$. From (A-29), 


$$
h_{r \varphi}^{\prime}-\frac{\lambda^{2} r}{2} h_{r \varphi}=-\left(h_{t \varphi}^{\prime}+\frac{\lambda^{2} r}{2} h_{t \varphi}\right)
$$

This first order differential equation for $h_{r \varphi}$ can be readily solved, albeit that the resulting analytical expression from the generic solution

$$
\begin{aligned}
& h_{r \varphi}(\lambda r)=\frac{1}{p(\lambda r)} \int p(\lambda r) f(\lambda r) d(\lambda r), \text { in which } \\
& f(\lambda r)=\frac{\partial}{\partial(\lambda r)} h_{t \varphi}(\lambda r)+\frac{\lambda r}{2} h_{t \varphi}(\lambda r) ; p(\lambda r)=\exp \left\{\int-\frac{\lambda r}{2} d(\lambda r)\right\}=\exp \left(-\frac{\lambda^{2} r^{2}}{4}\right),
\end{aligned}
$$

is a rather complicated one.

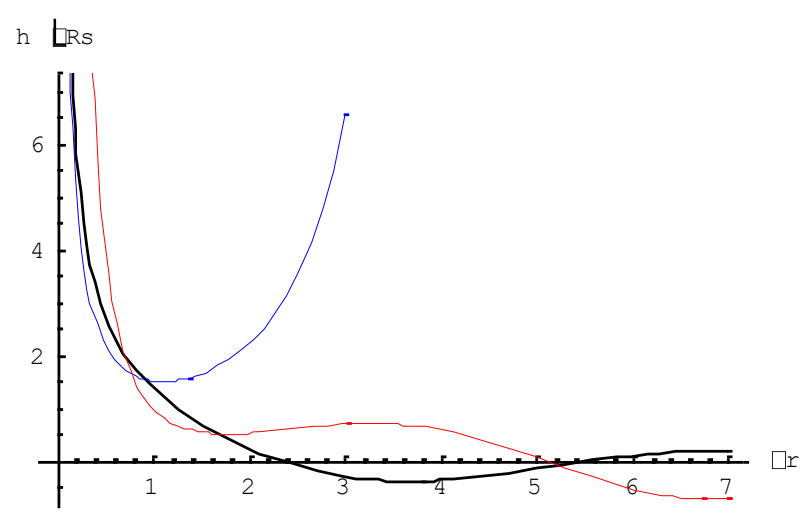

Fig. A-1: Relative values of the metric quantities $h_{t \varphi}$ (black) and $-h_{r \varphi}$ (blue) as a function of $\lambda r$. The red curve represents the function in the right-hand part of (A-36).

Figure A-1 illustrates the behavior of the calculated $-h_{r \varphi}$ as a function of $\lambda r$ compared with $h_{t \varphi}$. From (A-36), it is obvious that if $\lambda r \rightarrow 0, h_{r \varphi} \approx-h_{t \varphi}$. The vertical axis is normalized to a dimensionless quantity, by writing, under consideration of (A-30) and (A-33),

$h_{t \varphi}=\frac{2}{c^{2}} \frac{G M}{r}\{\cos \lambda r+\sin \lambda r\}=\frac{\lambda R_{S}}{\lambda r} \cos \lambda r+\sin \lambda r ; R_{S}=\frac{2 M G}{c^{2}}$.

Note that $R_{S}$ is the Schwarzschild radius of the cosmological system (with central force) under consideration. The black curve shows the normalized value of $h_{t \varphi}$. It is gradually decreasing by $r^{-1}$. The blue curve shows the normalized value of $h_{r \varphi}$ as calculated from the differential equation (A-36). This quantity tend to explode with increasing $\lambda r$. Nevertheless the functions on both sides of (A-36) remain the same and show the gradual finite behavior, shown by the red curve. The reason is due to sign differences between the left-hand part and the right-hand part of (A-35). Subtraction of two large quantities makes the result still small enough. Nevertheless, the exponential increase of $h_{r \varphi}$ may violate the linearization 
approximation. This requires proper investigation. Because eventually (for relatively large $\lambda r) h_{r \varphi} \gg h_{t \varphi}$, and considering that $2 \Lambda=\lambda^{2}$, we may reformulate (A-35) as,

$\frac{1}{\lambda r}>>\frac{\lambda}{8} R_{S}\left(\frac{h_{r \varphi}}{\lambda R_{S}}\right)$

Hence, the cross over value $\lambda r_{0}$ is determined as,

$\lambda r_{0}=\frac{8}{h_{r \varphi}\left(r_{0}\right)}=\frac{8}{\lambda R_{S} h_{r \varphi}\left(\lambda r_{0}\right)}$

where $h_{r \varphi}(\lambda r)$ is given by (A-37). From (A-39) it is obvious that as long as $h_{r \varphi}<1$, the upper limit is far beyond $\lambda r=8$. However, because of the exponential growth of $h_{r \varphi} / \lambda R_{S}$ with $\lambda r$, the limit can be shifted near to this limit or even shifted below. This may spoil the weak field limit assumption. Hence, the actual validity range of the linearization heavily depends on the value of the product $\lambda R_{S}$. Once this product is known, the cross-over value of $\lambda r_{0}$ and the associated value of the metric component $h_{r \varphi}$ can be calculated from the known curve $h_{r \varphi} / \lambda R_{S}$ shown in fig. A-1. Because of its exponential growth, the upper limit for $\lambda r$ that justifies the linearization approximation, is below, but probably near, to the cross-over value.

The assessment of a meaningful quantitative value to the product $\lambda R_{S}$ is possible by invoking the value of Einstein's $\Lambda$ for cosmological systems with a central mass. As shown in the main text, this is obtained by the application of the theory to Milgrom's MOND, expressed by eq. (26). This expression relates Einstein's Cosmological Constant $\Lambda$ with Milgrom's acceleration constant $a_{0}$ as,

$2 \Lambda=\lambda^{2}=\frac{2 a_{0}}{5 M G}$.

Hence,

$\lambda R_{S}=2 \sqrt{\frac{a_{0}}{a_{L}} \frac{R_{S}}{L}} ; a_{L}=\frac{c^{2}}{L}$,

where $a_{L}$ is the gravitational acceleration constant at distance $L$ from the centre of the cosmological system under consideration. Choosing $L$ as the Hubble range $L=c t_{H}$ and defining $c^{2} / L=a_{L}$ as the acceleration at the verge of the Hubble range, and considering that Milgrom's acceleration constant amounts to $a_{0} \approx 1.25 \times 10^{-10} \mathrm{~m} / \mathrm{s}^{2}$, the ratio $a_{L} / a_{0}$ amounts to, $a_{L} / a_{0} \approx 6.9 / 1.25=5.52$. The Schwarzschild radius $R_{S}$ of a typical galaxy, like the Milky Way, is about 0.2 lightyear, while the Hubble time amounts to $t_{H} \approx 13.5$ Gyear. Hence, typically 
$\lambda R_{S} \approx 3.3 \times 10^{-6}$.

From (A-42), (A-40) and (A-37), it is found that the cross-over $\lambda r_{0}$ amounts to

$\lambda r_{0} \approx 7.25$

The associated value of the metric component amounts to $h_{r \varphi}=1.01$. That violates the weak field approximation. However, at $\lambda r=6$, the metric component is drops to $h_{r \varphi}=0.017$. Hence, It is fair to say that, up to a normalized spatial distance near to $\lambda r_{0} \approx 6$, the derived gravitational wave equation (A-31) for galaxies akin to the Milky Way maintains its validity.

The remaining issues: (b) the other two equations

Before appearance of the massive source, we have for $(A-3 c)$,

$\left\{1+\frac{r}{2 g_{r r}}\left(\frac{g_{r r}^{\prime}}{g_{r r}}-\frac{g_{t t}^{\prime}}{g_{t t}}\right)-\frac{1}{g_{r r}}\right\}-g_{\vartheta \vartheta} \Lambda=$ background matter,

where $g_{\mu \mu}=\left(1,1, r^{2} \sin ^{2} \vartheta, r^{2}\right)$

After appearance of the massive source, we have

$1+\frac{r}{2 g_{r r}}\left(\frac{g_{r r}^{\prime}}{g_{r r}}-\frac{g_{t t}^{\prime}}{g_{t t}}\right)-\frac{1}{g_{r r}}-g_{\vartheta \vartheta} \Lambda=$ background matter plus source,

where $g_{\mu \mu} \neq\left(1,1, r^{2} \sin ^{2} \vartheta, r^{2}\right)$.

Due to the change of curving by the source, we have,

$1+\frac{r}{2 g_{r r}}\left(\frac{g_{r r}^{\prime}}{g_{r r}}-\frac{g_{t t}^{\prime}}{g_{t t}}\right)-\frac{1}{g_{r r}}-\left(g_{\vartheta \vartheta}-r^{2}\right) \Lambda=0$.

Under the constraint of the weak field limit, this equation can be rewritten as,

$\Lambda g_{\vartheta \vartheta}=\Lambda r^{2}-r h_{t \varphi}^{\prime}-h_{t \varphi}+\frac{r}{2}\left(h_{r \varphi}^{\prime}+h_{t \varphi}^{\prime}\right)+\left(h_{r \varphi}+h_{t \varphi}\right)=0$

As long as $\Lambda g_{\vartheta \vartheta}$ is close to $\Lambda r^{2}$, the metric (A-1) maintains it validity. This is true as long as

$$
\begin{aligned}
& r^{2}>>\frac{1}{\Lambda}\left(r h_{t \varphi}^{\prime}+h_{t \varphi}\right), \text { and } \\
& r^{2}>>\frac{1}{\Lambda}\left\{\frac{r}{2}\left(h_{r \varphi}^{\prime}+h_{t \varphi}^{\prime}\right)+\left(h_{r \varphi}+h_{t \varphi}\right)\right\} .
\end{aligned}
$$


The split into two conditions is made for ease of analysis. Under consideration of (A-29), (A48b) can be rewritten as,

$$
r^{2}>>\frac{1}{\Lambda}\left\{\left(1+\frac{\lambda^{2} r^{2}}{2}\right) h_{r \varphi}+\left(1-\frac{\lambda^{2} r^{2}}{2}\right) h_{t \varphi}\right\} .
$$

Because with increasing $\lambda r$ the quantity $h_{r \varphi}$ is dominating over $h_{t \varphi},(\mathrm{A}-50)$ can be replaced by,

$$
r^{2}>>\frac{1}{\Lambda}\left(\frac{\lambda^{2} r^{2}}{2}\right) h_{r \varphi} \rightarrow r^{2}>>r^{2} h_{r \varphi} \rightarrow h_{r \varphi}<<1
$$

thereby concluding that the condition $(A-49 b)$ is covered by the weak field constraint.

Now we have established the upper spatial limit justified the linearization condition and concluded that condition ( $\mathrm{A}-48 \mathrm{~b}$ ) is covered by the weak field constraint, we are left with a single issue. That is condition (A-48a). Considering that,

$$
\begin{aligned}
& h_{t \varphi}=\frac{2}{c^{2}} \frac{G M}{r}\{\cos \lambda r+\sin \lambda r\} ; h_{t \varphi}^{\prime}=-\frac{R_{S}}{r^{2}}\{\cos \lambda r+\sin \lambda r\}+\frac{R_{S}}{r}(-\lambda \sin \lambda r+\lambda \cos \lambda r) \\
& h_{t \varphi}^{\prime}=-\frac{R_{S}}{r^{2}}\{(\lambda r-1) \cos \lambda r-(1+\lambda r) \sin \lambda r\},
\end{aligned}
$$

we have for (A-49a),

$$
\begin{aligned}
& r^{2}>>\frac{R_{S}}{\Lambda}(-\lambda \sin \lambda r+\lambda \cos \lambda r) \rightarrow \\
& r^{2}>>\frac{2 R_{S}}{\lambda}(-\sin \lambda r+\cos \lambda r) \approx r^{2}+\frac{2\left|R_{S}\right|}{\lambda}=r^{2}+\frac{2\left|R_{S}\right|}{\sqrt{2 a_{0}}} c \sqrt{5 M G / c^{2}}= \\
& r^{2}>>\frac{2\left|R_{S}\right|}{\sqrt{2 a_{0}}} c \sqrt{\frac{5 R_{S}}{2}}=\frac{2\left|R_{S}\right|}{\sqrt{2 a_{0}}} \sqrt{\frac{c^{2}}{L}} \sqrt{\frac{5 R_{S}}{2}} \sqrt{L}=2\left|R_{S}\right| \sqrt{\frac{a_{L}}{a_{0}}} \sqrt{\frac{5 R_{S} L}{2}} .
\end{aligned}
$$

Hence,

$r>>R_{G}$, where $R_{G}=\left(2\left|R_{S}\right| \sqrt{\frac{a_{L}}{a_{0}}} \sqrt{\frac{5 R_{S} L}{2}}\right)^{1 / 2}$.

As already noted, the Schwarzschild radius $R_{S}$ of a typical galaxy, like the Milky Way, is about 0.2 lightyear. For such a galaxy, the range $R_{G}$ calculated from (A-54) appears being $R_{G} \approx 278$ lightyear. Considering that the radius of the Milky Way is estimated as $100.000-180.000$ lightyear and that our solar system is at about 26.000 lightyear from the center, it will be clear that the wave equation (A-31) holds for the major part of the galaxy, thereby solving the anomaly problem of the stellar rotation problem. 
Table A1: metric tensor and Ricci tensor

\begin{tabular}{|l|l|}
\hline metric tensor & Ricci tensor \\
\hline$g_{t t} \equiv g_{00}$ & $R_{t t}=-\frac{1}{2} \frac{g_{t t}^{\prime \prime}}{g_{r r}}-\frac{\ddot{g}_{r r}}{2 c^{2} g_{r r}}+\frac{g_{t t}^{\prime}}{4 g_{r r}}\left(\frac{g_{r r}^{\prime}}{g_{r r}}+\frac{g_{t t}^{\prime}}{g_{t t}}\right)-\frac{\dot{g}_{r r}}{4 c^{2} g_{r r}}\left(\frac{\dot{g}_{r r}}{g_{r r}}+\frac{\dot{g}_{t t}}{g_{t t}}\right)-\frac{1}{r} \frac{g_{t t}^{\prime}}{g_{r r}}$ \\
\hline$g_{r r} \equiv g_{11}$ & $R_{r r}=-\frac{1}{2} \frac{g_{t t}^{\prime \prime}}{g_{t t}}-\frac{\ddot{g}_{r r}}{2 c^{2} g_{t t}}+\frac{g_{t t}^{\prime}}{4 g_{t t}}\left(\frac{g_{r r}^{\prime}}{g_{r r}}+\frac{g_{t t}^{\prime}}{g_{t t}}\right)-\frac{\dot{g}_{r r}}{4 c^{2} g_{t t}}\left(\frac{\dot{g}_{r r}}{g_{r r}}+\frac{\dot{g}_{t t}}{g_{t t}}\right)+\frac{1}{r} \frac{g_{r r}^{\prime}}{g_{r r}}$ \\
\hline$g_{\vartheta \vartheta} \equiv g_{22}=r^{2}$ & $R_{\vartheta \vartheta}=1+\frac{r}{2 g_{r r}}\left(\frac{g_{r r}^{\prime}}{g_{r r}}-\frac{g_{t t}^{\prime}}{g_{t t}}\right)-\frac{1}{g_{r r}}$ \\
\hline$g_{\varphi \varphi} \equiv g_{33}=r^{2} \sin ^{2}(\vartheta)$ & $R_{\kappa \varphi}=\sin ^{2}(\vartheta) R_{\vartheta \vartheta}$ \\
\hline
\end{tabular}

\section{References}

[1] ] D. Griffiths, Introduction to Elementary Particles, ISBN 3527406018, Wiley (2008)

]2] J.A. Frieman, M.S. Turner, D. Huterer, Dark energy and the accelerating universe, Ann.

Rev. Astronomy and Astrophys. 46, 385 (2008)

[3] P.J.E. Peebles, B. Ratra, Bharat (2003), The cosmological constant and dark energy, Reviews of Modern Physics. 75 (2): 559, (2003)

[4] J. Schwichtenberg, Demystifying Symmetry Breaking, http//:jacobschwichtenberg.com, Aug.20, 2020

[5] Blanchet, L, Dipolar particles in general relativity, Class.Quant.Grav.24, 14, 3541(2007)

[6] Blanchet, L. and Tiec, A., Dipolar dark matter and dark energy, Phys.Rev.D80, 023524 (2009)

[7] D. Hajdukovic, Is dark matter an illusion created by the gravitational polarization of of the quantum vacuum?, Astrophysics and Space Science, 334, vol.2, 215 (2011)

[8] A. Raymond Penner, Gravitational anti-screening as an alternative to the $\Lambda$ CDM model, Astrophys. Space Sci. 361:124 (2016)

[9] E. Roza, Astrophys. And Space Sci., The vacuum polarization paradox and its solution, 364:73, doi.org/10.1007/s10509-019-3561-9 (2019)

[10] P. Debye and E. Huckel, Physik. Zeitschrift, Zur Theorie der Elektrolyte, vol. 24, 9, 185 (1923)

[11] F. Englert, R. Brout, Broken symmetry and the mass of gauge vector bosons, Phys. Rev. Lett. 13 (9), 321 (1964)

[12] P. Higgs, Broken symmetries and the masses of gauge bosons, Phys. Rev. Lett. 13 (16), 509 (1964)

[13] A.L. Fitzpartrick, K.M. Zurek, Non-relativistic effective theory of dark matter, Phys.

Rev.D 82, 075004 (2010)

[14] C.M. Ho, R.J. Scherrer, Anapole dark matter, Phys. Lett. B 722, 341 (2013) 
[15] E. Majorana, Teoria simmetrica dell'ellettrone e del positrone, Nuovo Cimento, 14, 171 (1937)

[16] E. Roza, On the second dipole moment of Dirac's particle, Found. of Phys. 50, 828 (2020)

[17] P.A.M. Dirac, The quantum theory of the electron, Proc.Royal Soc. London, A 117, 610 (1928)

[18] J.D. Bjorken, S.D. Drell, Relativistic Quantum Mechanics, McGraw-Hill Book Cie (1964)

[19] S. Weinberg, Gravitation and Cosmology, John Wiley \& Sons, Inc., New York (1972)

[20] A. Einstein, Relativity: The Special and General Theory, H. Holt and Company, New York

[21] T.A. Moore, A General Relativity Workbook, University Science Books, (2013)

[22] B. Schutz, A First Course in General Relativity, $2^{\text {nd }}$ ed, Cambridge Univ. Press, New York (2009)

[23] D. Perkins, Introduction to High Energy Physics, $4^{\text {th }}$ Ed., Cambridge Univ. Press, Cambridge UK (2000)

[24] F. Kottler, Über die physikalischen Grundlagen de Einsteinsch Gravitationstheorie, Ann. Physik 56, 361, 401 (1918)

[25] H. Weyl, Über die statischen kugelsymmetrischen Lösungen von Einsteins

"kosmologischen" Gravitationsgleichungen, Phys. Z. 20, 31 (1919)

[26] E. Trefftz, Das statische Gravitationsfeld zweier Massenpunkte in der Einsteinschen Theorie, Mathem. Ann. 86, 317 (1922)

[27] Li-Feng Sun et al., Schwarzschid-de Sitter metric and inertial Beltrami coordinates, Modern Phys. Lett. A 28, 1350114 (2013)

[28] A. Einstein, Preuss. Akad. Wiss, Berlin (Math. Phys.), Cosmological considerations in the General Theory of Relativity, 142 (1917)

[29] www.scholarpedia.org/article/Cosmological constant, Accessed Sept. 2020

[30] S. Carroll, W. Press and E. Turner, The cosmological constant, Ann. Rev. Astronomy and Astrophys, 30, 499 (1992)

[31] E. Roza, On the symmetry of the universe, www.preprints.org, doi:10.20944/preprints202007.0736.v2

[32] A. Einstein, Principielles zur allgemeinen Relativitätstheorie, , Ann. der Physik, 4 (55), $241(1918)$

[33] C.A. Gonano, R.E. Zich, M. Mussetta, Definition for polarization P and magnetization M fully consistent with Maxwell's equations, Progr. in Electromagn. Res. B, 64, 83 (2015)

[34] https://en.wikipedia.org/wiki/Polarization density, accessed Oct., 2020

[35] E. Verlinde, On the origin of gravity and the laws of Newton, JHEP 1104, 029 ; arXiv: 1001.0785v1 [hep-th] (2011)

[36] E. Verlinde, Emergent gravity and the dark universe, SciPost Phys. 2, 016 (2017); arXiv: 1611.02269v2 [hep-th] (2016)

[37] R.K. Pathria, The universe as a black hole, Nature, $240(5379,298$ (1972)

[38] Weinberg, S. : A model of leptons, Phys. Rev. Lett 19 (21), 1264, doi:10.1103/PhysRevLett.191264W (1967)

[39] Nambu, Y., Jona-Lasinio, G: Dynamic model of elementary particles based on an analogy with superconductivity, Phys. Rev. 124(1) 246 (1961)

[40] Wilczek, F.: Hard-core revelations, Nature (London), 445, 156 (2007)

[41] Roza, E.: A hypotherical H-particle, Phys. Essays, 24, 1, 72 (2011)

[42] Roza, E.: The H-type quark and the mass of mesons, Phys. Essays, 25, 2, 191 (2012)

[43] E. Roza, The gravitational constant as a quantummechanical expression, Results in Physics, 6, 149 (2016) 
[44] E.Roza, On the quark scaling theorem and the polarisable dipole of a quark, www.preprints.org, doi: $10.20944 /$ preprints202006.0304.v1

[45] S. Wolfram, Mathematica, Addison Wesley, Redwood City A (1991)

[46] Whitten, R.C., Smith, F.T.: Symmetric Representation for Three-Body Problems. II.

Motion in Space, J. Math. Phys. 9, 1103 (1968)

[47] Bartlett, P., Howard, B.J.: The rotational-vibrational spectrum of symmetric non-rigid triatomics in hyperspherical coordinates: the $\mathrm{H}^{+}{ }_{3}$ molecule Molecular Physics, Vol. 70, No. 6, 1001 (1990)

[48] Roza, E., The H-type quark and the baryons, Phys. Essays, 27, 3, 390 (2014)

[49] M. Tanabashi et al. (Particle Data Group), Review of Particle Physics, Phys. Rev. D 98 (3), 1 (2018) 